\title{
The BMP2/4 ortholog Dpp functions as an inter-organ signal that regulates developmental timing in Drosophila
}

Linda Setiawan, Alexis L. Woods, Iswar K. Hariharan*

Department of Molecular and Cell Biology

University of California, Berkeley

361 LSA

Berkeley CA 94720-3200

*Correspondence to: ikh@berkeley.edu 


\begin{abstract}
:
For many organisms, developmental transitions are triggered by a neuroendocrine axis and are contingent upon multiple organs achieving sufficient growth and maturation. How the status of peripheral organs is communicated to the neuroendocrine axis is not known. In Drosophila larvae, metamorphosis is triggered by the steroid hormone ecdysone, secreted by the prothoracic gland (PG). Here we show that the BMP2/4 ortholog Dpp, which regulates growth and patterning of larval imaginal discs, also functions as a systemic signal to regulate developmental timing. Dpp from peripheral tissues, mostly imaginal discs, can reach the PG and inhibit ecdysone biosynthesis. As the discs grow, Dpp signaling decreases in the PG, thus alleviating the inhibition of ecdysone biosynthesis, and permitting entry into metamorphosis. We suggest that if a tissue can trap more morphogen locally as it grows and matures, then circulating levels of morphogen can provide a systemic readout of organ size and maturation.
\end{abstract}

One Sentence Summary:

Dpp functions as a long-range endocrine signal between peripheral tissues and the prothoracic gland to regulate developmental timing in Drosophila. 
Organismal development is often orchestrated by a neuroendocrine axis, such as the hypothalamic-pituitary axis in mammals. Mechanisms likely exist by which the growth and maturation of peripheral organs are monitored by the neuroendocrine axis prior to important developmental transitions. These mechanisms remain undefined. The onset of metamorphosis in Drosophila, a dramatic developmental transition, results from a steep increase in the level of the steroid hormone ecdysone (1) made by the prothoracic gland (PG), a part of the ring gland (Fig. 1A). The onset of pupariation in larvae is contingent upon sufficient growth (2), notably of the imaginal discs (3), the precursors of adult structures such as the wing. Regulators of ecdysone production in the PG include the peptide prothoracicotropic hormone (PTTH) (4), insulin signaling $(5,6)$, the activin pathway $(7)$, the bantam (ban) microRNA $(8)$, and under conditions of starvation, circulating Hedgehog made by enterocytes (9). It is not understood how growth of peripheral tissues can influence these pathways. The insulin/relaxin family member Dilp8, secreted by damaged or overgrown imaginal discs, delays pupariation $(10,11)$, but its role in regulating developmental timing under normal conditions is unclear.

The BMP2/4 ortholog, Dpp, functions as a morphogen to regulate growth and patterning within many tissues including imaginal discs (12). We increased $d p p$ expression briefly during the early third larval instar (L3) using $r n-G a l 4$ and a temperature-sensitive repressor, Gal80 ${ }^{\text {ts }}$ (hereafter $r n^{t s}>d p p$ ) (Figure 1B). $r n$-Gal4 is expressed in wing discs and also some other tissues (Fig. S1A). $r n^{t s}>d p p$ did not increase wing disc size (Figure 1C) or adult wing size (not shown) but markedly delayed pupariation (Fig. 1B) resulting in larger pupae, likely due to an extended growth phase (Fig. 1D). Surprisingly, an activated form of the Dpp receptor Thickveins ( $\left.T k^{Q D}\right)(13)$, $\left(r n^{t s}>t k v^{Q D}\right)$, which functions cell autonomously, elicited only a modest delay (Fig. 1B-C). Expression of $d p p$, but not $t k v^{Q D}$, increased Dpp signaling beyond the wing pouch (Fig. 1E-G, arrowheads in Fig. 1F, G) and in the PG, as assessed by increased nuclear pMad (Fig. 1E'-G'). rnGal4 is not expressed in the ring gland but is expressed in the central nervous system, CNS (Fig. S1A). Delayed pupariation, nuclear localization of pMad in the PG, and increased pupal size all still occurred when neuronal expression of $r n$-Gal4 was prevented (Fig. S1B-I). Thus, the Dpp that reaches the PG in these experiments is likely produced by more distant non-neuronal cells. 
Indeed, $d p p$ expressed in various peripheral tissues delays pupariation (Fig. S2A-G) suggesting effects of Dpp outside its tissue of origin.

Did $d p p$ delay pupariation indirectly via Dilp8 production? $r n^{t s}>d p p$ or $r n^{t s}>t k v^{Q D}$ caused little cell death or dilp8 expression (Fig. S3A-F) and $r n^{t s}>d p p$ delayed pupariation more than $r n^{t s}>$ dilp8 (Fig. 1B) suggesting that $d p p$ does not function upstream of dilp8. Conversely, $r n^{t s}>d i l p 8$ did not affect nuclear pMAD in the wing disc or PG (Fig. $\left.1 \mathrm{H}, \mathrm{H}^{\prime}\right)$ indicating that Dilp8 does not activate Dpp signaling. Most importantly, $r n^{t s}>d p p$ delayed pupariation in a dilp8 mutant implying that Dpp functions independently of dilp8 (Fig. S3G). Finally, expressing $t k v^{Q D}$ using the ring gland driver P0206-Gal4 (5) delayed pupariation and increased pupal mass (Fig. 1I-K). Pupariation was delayed using a PG-specific driver, but not using drivers expressed in either the corpus allatum (CA) or corpora cardiaca (CC) (Fig. S4A-B). Conversely, overexpression of brinker (brk) in the PG, which represses many Dpp target genes, arrested larvae in L2, yet caused wandering (prepupal) behavior, similar to the precocious metamorphosis observed with increased Activin signaling (7) (Fig. S4C-N). Thus, Dpp signaling in the PG itself regulates the timing of developmental transitions.

Can Dpp reach the PG from peripheral tissues? When GFP-dpp (14) was expressed using rn-Gal4 $\left(r n^{t s}>\right.$ GFP-dpp), GFP and nuclear pMad were detected in the PG but not in the immediately adjacent CA (Fig. 2A-B) suggesting localization dependent on binding to specific receptors. A different secreted protein ANF-GFP (15) was not detected in the PG (Fig. 2C-D). A processed form of GFP-Dpp was detected in the hemolymph of $r n^{t s}>$ GFP-dpp larvae (Fig. 2E). Ex vivo, Dpp can diffuse from discs to the PG. Nuclear pMad was not observed in the PG portion of ring glands cultured alone (Fig. $2 \mathrm{~F}$ ) but was observed in ring glands co-cultured with $r n^{t s}>d p p$ wing discs, or with $r n^{t s}>d p p$ larval hemolymph (Fig. 2G, H). Thus, Dpp can diffuse from discs to the PG via the hemolymph. 
Neither inducing apoptosis in the wing disc, nor disc overgrowth in discs large ( $\mathrm{dlg}$ ) hemizygotes resulted in nuclear pMad in the PG but caused local Dilp8 production (Fig. S5) and delayed pupariation (not shown). Thus, unlike Dilp8, Dpp is not released by discs in response to tissue damage or overgrowth.

To address a role for Dpp signaling in the PG during normal development, we extended our analysis to include ring glands at earlier time points during the final larval stage (L3) by visualizing nuclear pMad (Fig. 3A-C) and two reporters, dad-RFP (Fig. 3D-I) and brk-GFP (Fig. 3DF, J-L). dad-RFP expression increases with Dpp signaling while brk-GFP expression increases as Dpp signaling decreases. In contrast to the PG in late L3 (120 h after egg lay, AEL), we observed nuclear pMad in the PG in early L3 (72 h AEL). As larvae progress through L3, Dpp signaling progressively decreases. By comparison, Dpp signaling was consistently high in the CC (Fig. 3AC) and consistently low in the CA (Fig. 3J-L); its function in the CA and CC is not known.

To help identify the source of Dpp that reaches the PG, we expressed UAS-dpp ${ }^{R N A i}$ using Gal4 drivers and examined pMad levels in the PG at $72 \mathrm{~h} \mathrm{AEL}$ when nuclear pMad is normally observed. In dpp-Gal4 UAS-dpp ${ }^{R N A i}$ larvae, the level of nuclear pMad is greatly reduced (Fig. 3MN). Thus, the Dpp must be produced by cells with current or past expression of $d p p-G a / 4$. $d p p$ Gal4 is expressed in some, but not all, Dpp-producing cells. G-TRACE (16) identifies both current (RFP) and previous (GFP) expression of $d p p$-Gal4 (Fig. 30-T); we detected expression in imaginal discs (Fig. 30, Fig. S6A-C), the salivary glands (Fig. 3P) and CNS (Fig. 3Q) but not in the gut (Fig. 3R), fat body (Fig. 3S), ring gland (Fig. 3T) (also see legend to Fig. S6) or lymph gland (Fig. S6D). $d p p$-Gal4 is expressed in the salivary glands (Fig. 3P) but $d p p$ itself is not, either in embryos (17) or in wandering L3 larvae (18). In addition to the optic lobes and parts of the ventral nerve cord, CNS dpp-Gal4 expression included the PTTH-expressing neurons which innervate the PG (Fig. $3 \mathrm{U}-\mathrm{V}$ ) and expression is blocked in the presence of elav-Gal80 (Fig. 3W). However, expression of GFP-dpp in PTTH-producing neurons does not reach the PG nor is nuclear pMad observed in PG cells when GFP-dpp is expressed using ptth-Gal4 (Fig. S6E-H). Additionally, expression of $d p p^{R N A i}$ 
either using elav-Gal4 or ptth-Gal4 does not reduce pMad expression in the PG (Fig. S6I-V), indicating that the Dpp is unlikely to be from the PTTH neurons or other neurons. Taken together, these experiments suggest that the Dpp that reaches the PG is mostly from the imaginal discs. Additionally, the ability of $d p p-G a / 4, U A S-d p p^{R N A i}$ to reduce pMad levels in the PG indicates that the pathway is indeed mostly being activated by Dpp and not other ligands such as Gbb (19).

We examined the expression of several ecdysone biosynthesis enzymes (20) in FLP-out clones with altered Dpp signaling (Fig. S7A). In early L3, when Dpp signaling is high, reducing Dpp signaling by overexpressing dad (21) increased expression of Disembodied (Dib) (Fig. 4A) or Shadow (Sad) (Fig. S7B). In late L3, with lower Dpp signaling, augmenting Dpp signaling with activated Tkv reduced expression of Dib (Fig. 4B), Sad, Spookier (Spok) and Phantom (Phm) (Fig. S7C-E). Delayed pupariation and increased pupal weight caused by increased Dpp signaling in the PG can be suppressed partially by providing the ecdysteroid 20-OH ecdysone (20E) (Fig. 4CD). In the PG, increased Dpp signaling increased bantam (ban) expression (Fig. 4E). Since ban expression delays pupariation and is negatively regulated by insulin signaling (8), Dpp and insulin signaling likely converge upstream of ban (Fig. S8A, B, D-N). Dpp signaling also functions antagonistically to the Activin pathway (Fig. S8C-E, O) as has been observed in the wing disc (22).

Dpp production, at least in wing discs, increases as discs grow during L3 (23). Dpp from discs can pass through the basement membrane into the hemolymph $(24,25)$. It is therefore surprising that Dpp signaling in the PG decreases concurrently. One possibility is that the PG becomes less sensitive to Dpp, perhaps by a downregulating Dpp receptors or other signaling components. However, pulses of Dpp in late $\mathrm{L} 3$ using $r n^{t s}>d p p$ elicit undiminished reporter responses in the PG (not shown). Although Dpp production in discs increases during L3 (23), disc growth results in a disproportionate increase in the number of cells that do not produce Dpp, yet can bind Dpp, thus expanding the "morphogen sink". Additionally, extracellular matrix 
proteins continue to be deposited on the basement membrane throughout L3 (26). For either or both of these reasons, as discs grow, more Dpp might be retained within the discs and less allowed to diffuse into the hemolymph and reach the PG (model in Fig. 4F). Any increase in hemolymph volume would further dilute Dpp in the hemolymph. Thus, growth and maturation of the larva can influence the timing of pupariation. Dpp therefore has dual roles: one within tissues to regulate patterning and growth and one in inter-organ communication to regulate developmental timing. It will be of interest to determine if vertebrate BMP proteins also have dual functions.

\section{REFERENCES AND NOTES}

1. N. Yamanaka, K. F. Rewitz, M. B. O'Connor, Ecdysone control of developmental transitions: lessons from Drosophila research. Annual review of entomology 58, 497-516 (2013).

2. L. Boulan, M. Milan, P. Leopold, The Systemic Control of Growth. Cold Spring Harb Perspect Biol 7, (2015).

3. B. C. Stieper, M. Kupershtok, M. V. Driscoll, A. W. Shingleton, Imaginal discs regulate developmental timing in Drosophila melanogaster. Dev Biol 321, 18-26 (2008).

4. K. F. Rewitz, N. Yamanaka, L. I. Gilbert, M. B. O'Connor, The insect neuropeptide PTTH activates receptor tyrosine kinase torso to initiate metamorphosis. Science 326, $1403-$ 1405 (2009).

5. J. Colombani et al., Antagonistic actions of ecdysone and insulins determine final size in Drosophila. Science 310, 667-670 (2005).

6. C. Mirth, J. W. Truman, L. M. Riddiford, The role of the prothoracic gland in determining critical weight for metamorphosis in Drosophila melanogaster. Curr Biol 15, 1796-1807 (2005).

7. Y. Y. Gibbens, J. T. Warren, L. I. Gilbert, M. B. O'Connor, Neuroendocrine regulation of Drosophila metamorphosis requires TGFbeta/Activin signaling. Development 138, 26932703 (2011).

8. L. Boulan, D. Martin, M. Milan, bantam miRNA promotes systemic growth by connecting insulin signaling and ecdysone production. Curr Biol 23, 473-478 (2013).

9. J. Rodenfels et al., Production of systemically circulating Hedgehog by the intestine couples nutrition to growth and development. Genes Dev 28, 2636-2651 (2014).

10. J. Colombani, D. S. Andersen, P. Leopold, Secreted peptide Dilp8 coordinates Drosophila tissue growth with developmental timing. Science 336, 582-585 (2012).

11. A. Garelli, A. M. Gontijo, V. Miguela, E. Caparros, M. Dominguez, Imaginal discs secrete insulin-like peptide 8 to mediate plasticity of growth and maturation. Science 336, 579582 (2012). 
12. F. Hamaratoglu, M. Affolter, G. Pyrowolakis, Dpp/BMP signaling in flies: from molecules to biology. Semin Cell Dev Biol 32, 128-136 (2014).

13. D. Nellen, R. Burke, G. Struhl, K. Basler, Direct and long-range action of a DPP morphogen gradient. Cell 85, 357-368 (1996).

14. E. V. Entchev, A. Schwabedissen, M. Gonzalez-Gaitan, Gradient formation of the TGFbeta homolog Dpp. Cell 103, 981-991 (2000).

15. S. Rao, C. Lang, E. S. Levitan, D. L. Deitcher, Visualization of neuropeptide expression, transport, and exocytosis in Drosophila melanogaster. J Neurobiol 49, 159-172 (2001).

16. C. J. Evans et al., G-TRACE: rapid Gal4-based cell lineage analysis in Drosophila. Nat Methods 6, 603-605 (2009).

17. R. D. St Johnston, W. M. Gelbart, Decapentaplegic transcripts are localized along the dorsal-ventral axis of the Drosophila embryo. EMBO J 6, 2785-2791 (1987).

18. J. B. Brown et al., Diversity and dynamics of the Drosophila transcriptome. Nature 512, 393-399 (2014).

19. T. E. Haerry, O. Khalsa, M. B. O'Connor, K. A. Wharton, Synergistic signaling by two BMP ligands through the SAX and TKV receptors controls wing growth and patterning in Drosophila. Development 125, 3977-3987 (1998).

20. Q. Ou, K. King-Jones, What goes up must come down: transcription factors have their say in making ecdysone pulses. Curr Top Dev Biol 103, 35-71 (2013).

21. K. Tsuneizumi et al., Daughters against dpp modulates dpp organizing activity in Drosophila wing development. Nature 389, 627-631 (1997).

22. A. J. Peterson, M. B. O'Connor, Activin receptor inhibition by Smad2 regulates Drosophila wing disc patterning through BMP-response elements. Development 140, 649-659 (2013).

23. O. Wartlick et al., Dynamics of Dpp signaling and proliferation control. Science 331, 1154-1159 (2011).

24. S. Harmansa, I. Alborelli, D. Bieli, E. Caussinus, M. Affolter, A nanobody-based toolset to investigate the role of protein localization and dispersal in Drosophila. eLife 6, (2017).

25. M. Ma, X. Cao, J. Dai, J. C. Pastor-Pareja, Basement Membrane Manipulation in Drosophila Wing Discs Affects Dpp Retention but Not Growth Mechanoregulation. Dev Cell 42, 97-106 e104 (2017).

26. J. C. Pastor-Pareja, T. Xu, Shaping cells and organs in Drosophila by opposing roles of fat body-secreted Collagen IV and perlecan. Dev Cell 21, 245-256 (2011).

\section{ACKNOWLEDGEMENTS}

We thank many colleagues in the fly community for constructive suggestions, María Domínguez, Marcos González-Gaitán, Pierre Léopold, Michael O’Connor, Lynn Riddiford, Chris

Rushlow, Michael Thomas Marr, Hilary Ashe and Konrad Basler for fly stocks and antibodies, the Bloomington, VDRC and TRiP stock centers, Octavio Bejarano for technical assistance and Jo Downes Bairzin, David Bilder, Robin Harris, Nipam Patel, Taryn Sumabat and Melanie Worley 
bioRxiv preprint doi: https://doi.org/10.1101/180562; this version posted August 24,2017 . The copyright holder for this preprint (which was not certified by peer review) is the author/funder. All rights reserved. No reuse allowed without permission.

for comments on the manuscript. IKH was funded by NIH grants R01GM085576 and

R35GM122490 and an American Cancer Society Research Professor Award (RP-16-238-06COUN). 

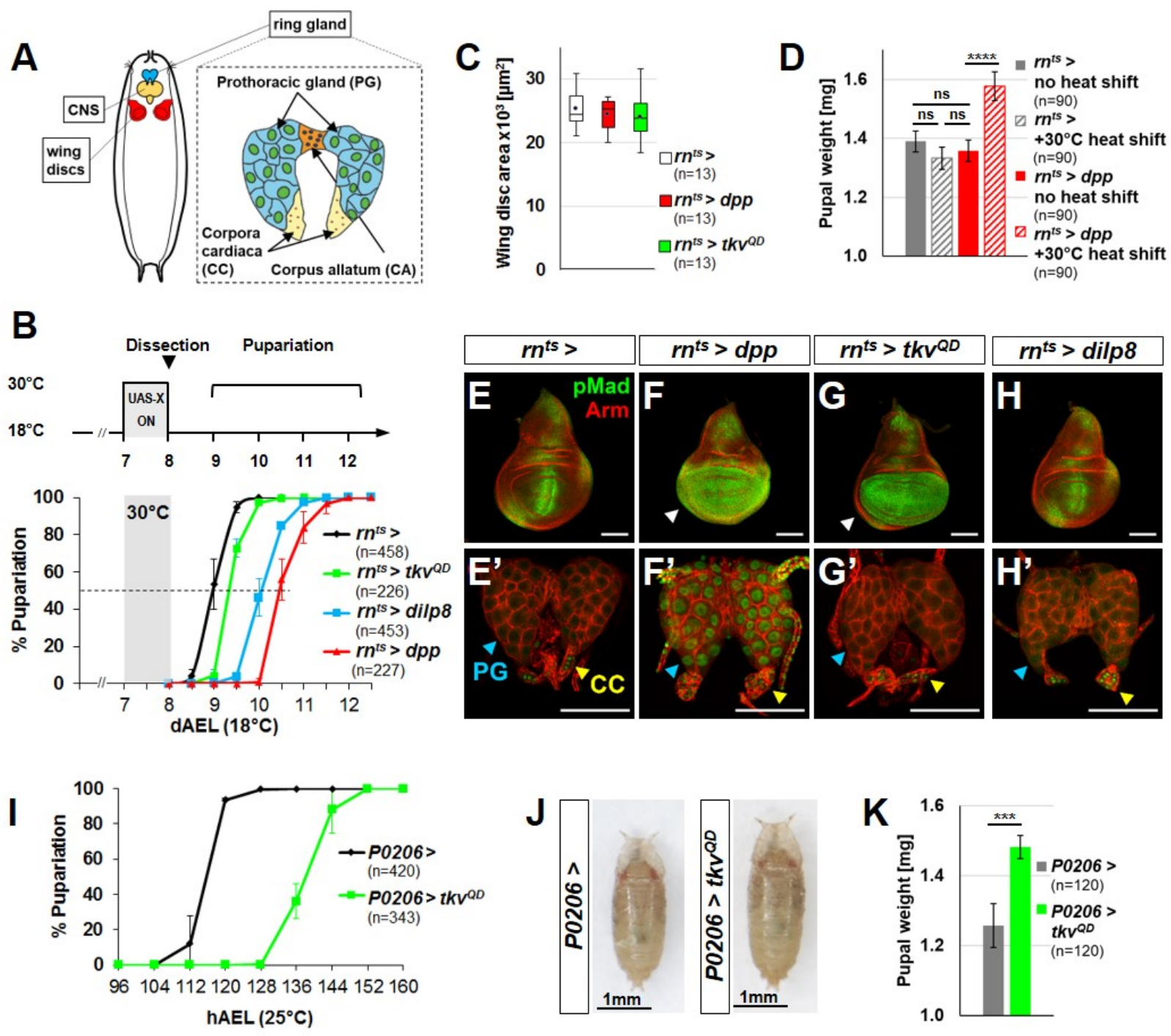

Figure 1 Expression of Dpp in peripheral tissues induces Dpp signaling in the PG and delays pupariation

(A) Schematic of ring gland (B) $r n^{t s}>d p p, r n^{t s}>t k v^{Q D}$, and $r n^{t s}>d i l p 8$ have a pupariation delay of $36.5 \pm 3.5 \mathrm{~h}, 9.5 \pm 3.0 \mathrm{~h}$, and $26.0 \pm 3.7 \mathrm{~h}$ respectively (C) wing disc areas (D) pupal weights (E-H) wing discs $\left(\mathbf{E}^{\prime}-\mathbf{H}^{\prime}\right)$ ring glands. Nuclear pMad is observed in the CC in all genotypes. (I) $P 0206>t k V^{Q D}$ has a $22.2 \pm 2.1 \mathrm{~h}$ developmental delay (J) pupal size (K) pupal weight increased in $P 0206>t k v^{Q D}$ by $18 \%$. Error bars indicate standard deviations, except $(\mathrm{C})$ which is a box and whiskers plot. $\mathrm{ns}=$ not significant; ${ }^{* * *} \mathrm{p}<0.001 ;{ }^{* * * *} \mathrm{p}<0.0001$. Scale bars $=100 \mu \mathrm{m}$. 


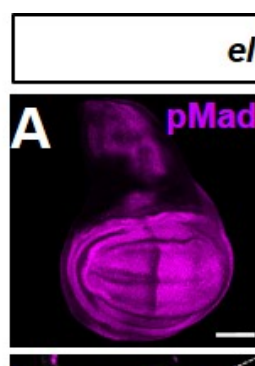

$e l a v^{80}, r n^{t s}>$ GFP-dpp
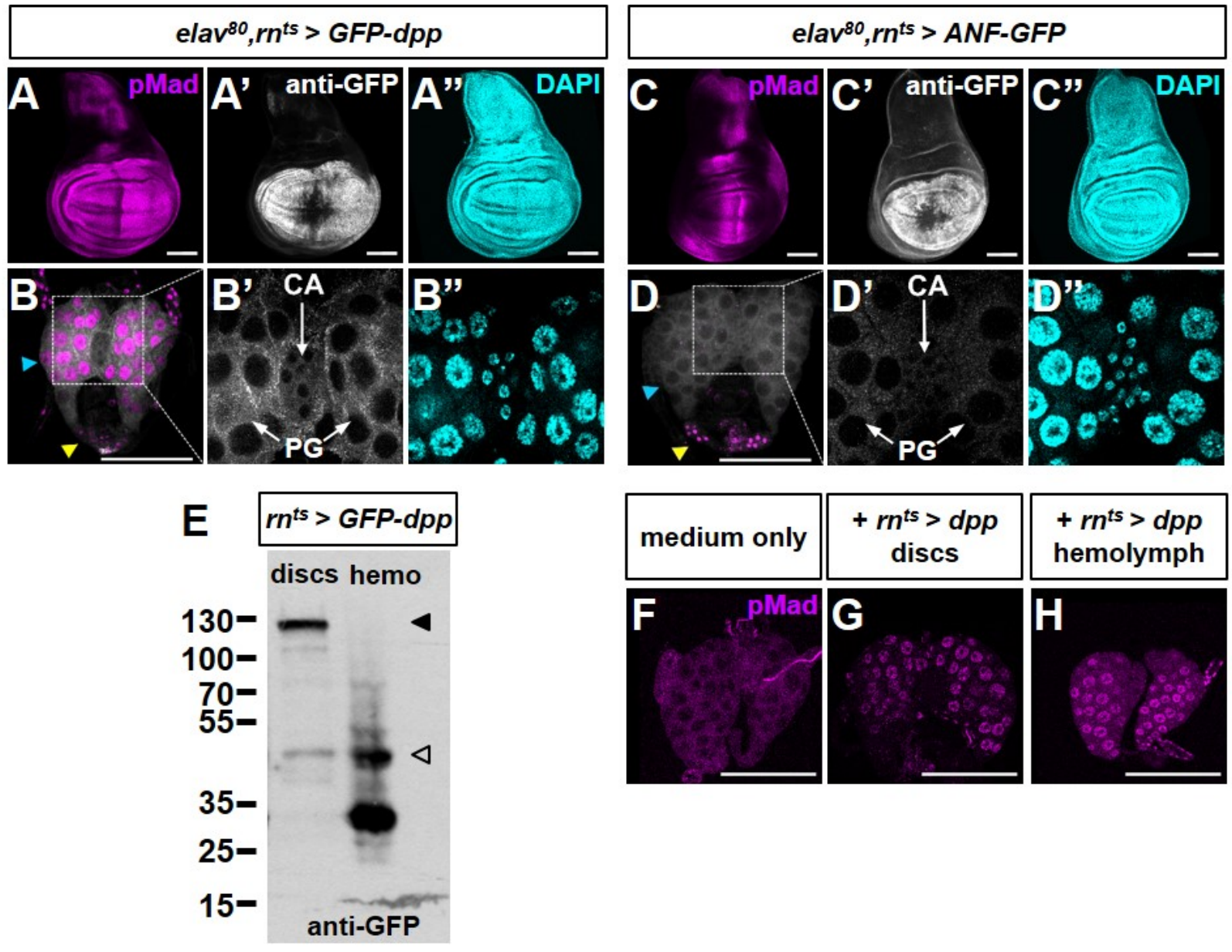

Figure 2 Dpp can diffuse from peripheral tissues to the PG via the hemolymph elav-Gal80 rn ${ }^{t s}>$ GFP-dpp wing disc (A-A") and ring gland (B-B'). Note GFP in PG but not CA (B'). Blue arrowhead PG, yellow arrowhead CC. (C-C", D-D") elav-Gal80 $r n^{\text {ts }}>$ ANF-GFP wing disc (C$\left.\mathbf{C}^{\prime \prime}\right)$ and ring gland (D-D"). (E) Western blot of $r n^{t s}>G F P$-dpp discs and hemolymph. Predicted unprocessed (filled arrowhead) and processed (unfilled arrowhead) forms. (F-H) Cultured ring glands. 

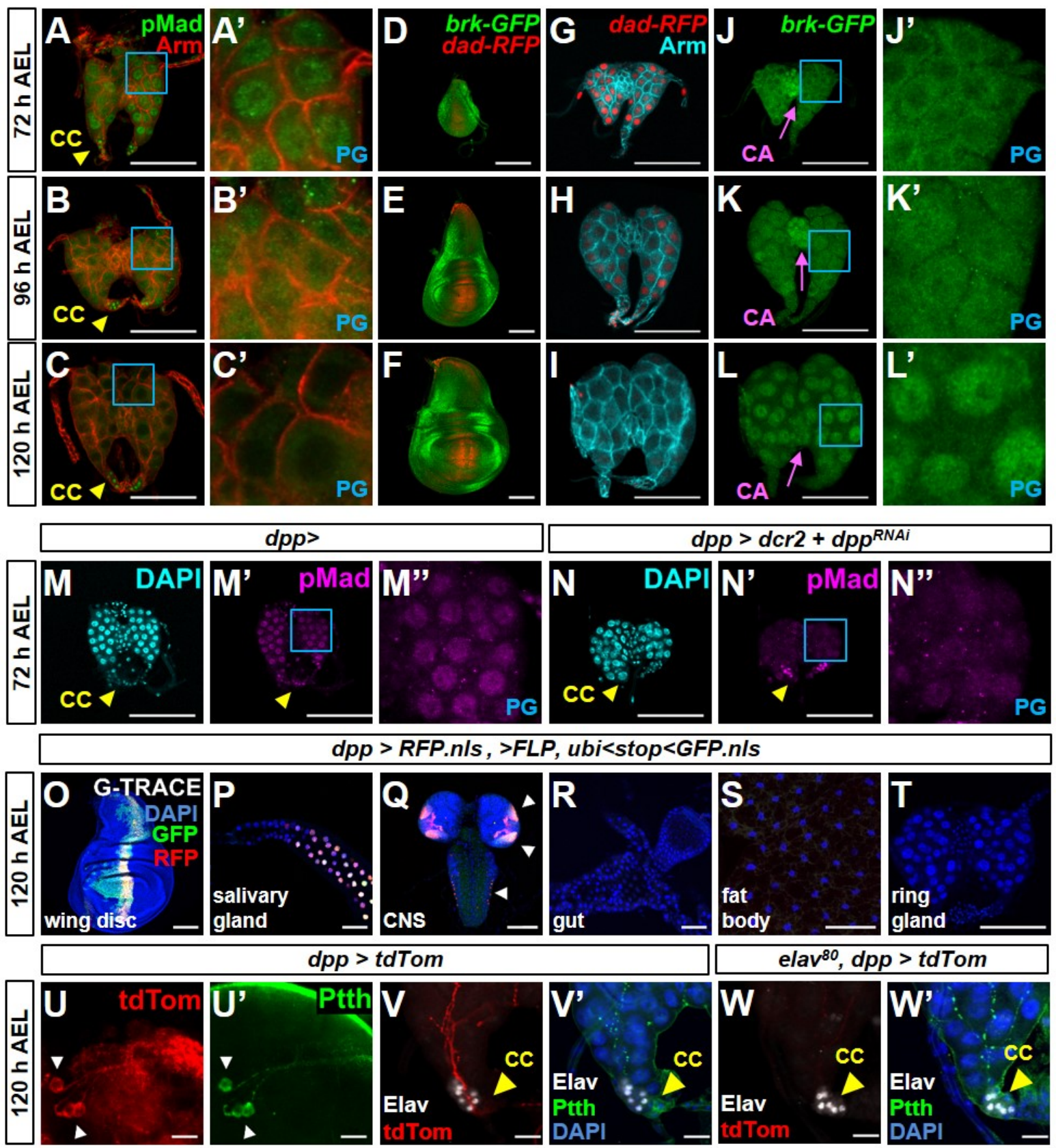

Figure 3 Dpp signaling in the PG decreases during L3

(A-L') Ring glands and wing discs, 72, 96 and $120 \mathrm{~h}$ after egg lay (AEL) at $25^{\circ} \mathrm{C}$. Boxed region in (A-C) at higher magnification in ( $\left.\mathbf{A}^{\prime}-\mathbf{C}^{\prime}\right)$. (D-F) Wing discs expressing dad-RFP and brk-GFP. (G-L') Ring glands with dad-RFP (G-I) or brk-GFP (J-L). Boxed regions at higher magnification in ( $\left.\mathrm{J}^{\prime}-\mathrm{L}^{\prime}\right)$. (M-N") Ring glands of indicated genotypes (O-T) dpp-Gal4 expression analyzed using G-TRACE. Current (RFP) or prior (GFP) expression. (U-V') Expression of dpp-Gal4 in the PTTH-secreting neurons showing cell bodies ( $\left.\mathbf{U}, \mathbf{U}^{\prime}\right)$ and axons that innervate the PG ( $\left.\mathbf{V}, \mathbf{V}^{\prime}\right)$. (W, $\left.\mathbf{W}^{\prime}\right) d p p-G a l 4$ expression in PTTH neurons blocked with elav-Gal80. Scale bars $100 \mu \mathrm{m}$ except (U-W') where they are $20 \mu \mathrm{m}$. 

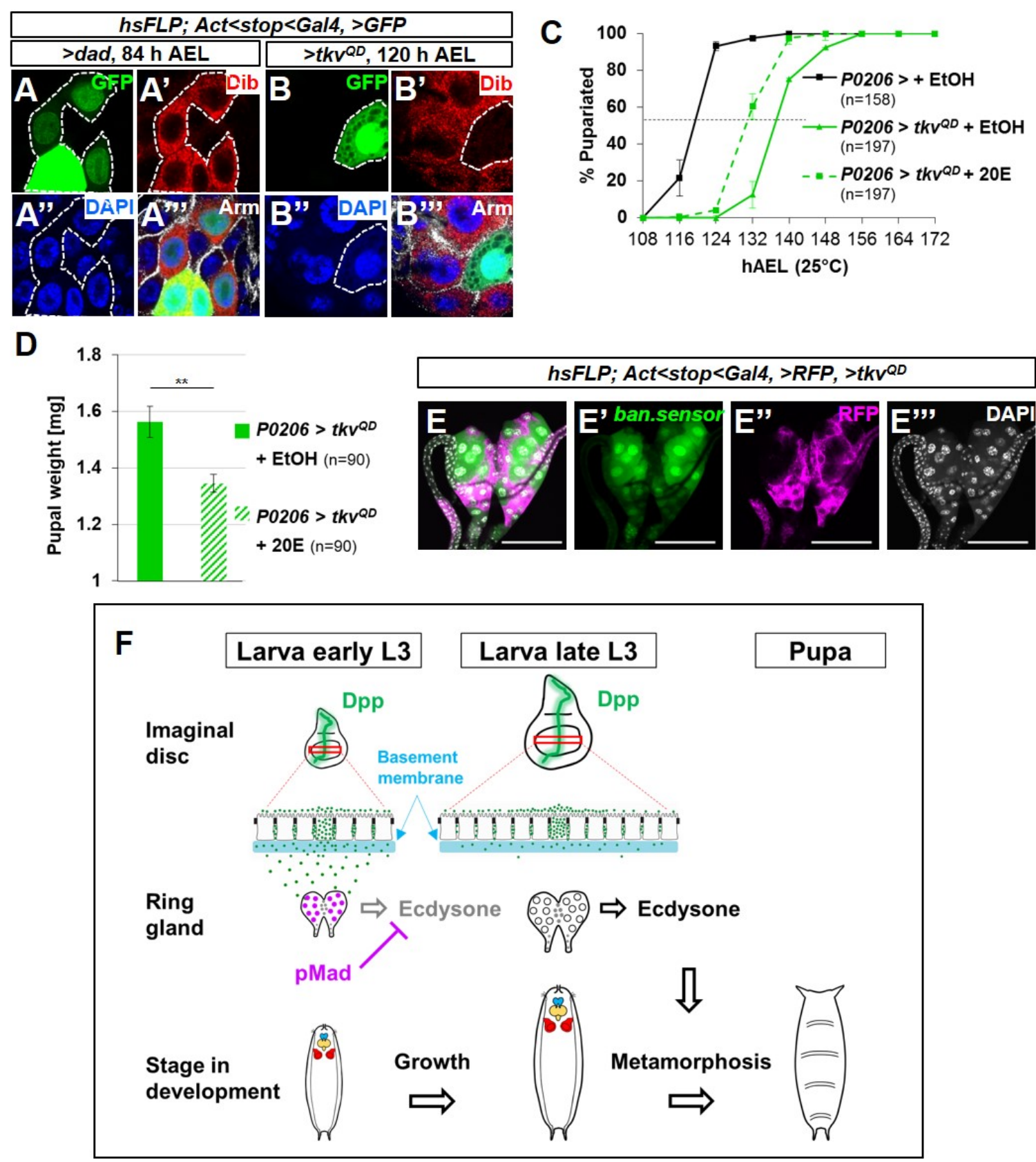

Figure 4 Dpp signaling in the PG represses ecdysone biosynthesis enzymes

(A-B'"') FLP-out clones induced 48hAEL, ring glands dissected $84 \mathrm{~h}$ AEL or $120 \mathrm{~h} \mathrm{AEL}$, examined for Disembodied (Dib) expression. (A-A"') GFP-expressing cells express UAS-dad, lowering Dpp signaling. (B-B'"') GFP-expressing cells express UAS-tkv ${ }^{Q D}$, increasing Dpp signaling. (C, D) Effect of feeding 20-hydroxyecdysone (20E) to $P 0206>t k v^{Q D}$ larvae. Mean pupariation times: P0206> +EtOH 119.0 $\pm 0.5 \mathrm{~h} ; P 0206>t k v^{Q D}+\mathrm{EtOH} 136.8 \pm 0.3 \mathrm{~h} ; P 0206>t k v^{Q D} 128.8 \pm 2.9 \mathrm{~h}$ (C) and pupal weight (D) ${ }^{* *} \mathrm{p}<0.01$. (E-E'”') Ring glands. FLP-out clones expressing UAS-tkv ${ }^{Q D}$ and RFP. Reduced ban sensor expression (green in $\mathbf{E}, \mathbf{E}^{\prime}$ ) indicates increased ban expression. Scale bars $100 \mu \mathrm{m}$. (F) Model for how discs influence Dpp signaling in the PG. 
bioRxiv preprint doi: https://doi.org/10.1101/180562; this version posted August 24,2017 . The copyright holder for this preprint (which was not certified by peer review) is the author/funder. All rights reserved. No reuse allowed without permission.

\section{SUPPLEMENTARY MATERIALS}

Materials and Methods

\section{Supplementary Figures S1-8}

References 1-37 


\section{SUPPLEMENTARY MATERIALS}

\section{The BMP2/4 ortholog Dpp functions as an inter-organ signal that regulates developmental timing in Drosophila}

Linda Setiawan, Alexis L. Woods, Iswar K. Hariharan

\section{Contents:}

Materials and Methods

Supplementary Figures S1-8

References 1-37 


\section{MATERIALS AND METHODS}

\section{Drosophila strains and husbandry}

Animals were raised on standard medium as used by the Bloomington Drosophila Stock Center. Fly stocks used are: $w^{1118}$ as wild-type control for all experiments, Oregon- $R, r n-G a l 4, t u b$ -

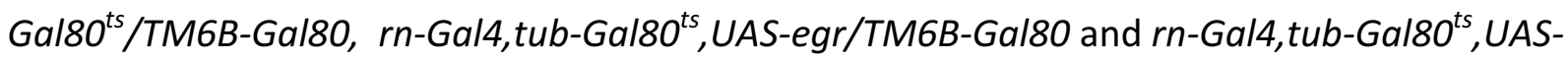
rpr/TM6B-Gal80 (27), UAS-dpp/TM6B (19), UAS-tkv Q253D/TM6B (13), dad-nRFP/CyO (23), UAS-GFPDpp (14), UAS-dilp8::3xFLAG (11), dpp-LG and lexOEGFP:dpp/TM6B (28), UAS-bantamA and bantam.sensor (29), P0206-Gal4, phm-Gal4 and ptth-Gal4 (gifts from Lynn Riddiford), UAS-dad and UAS-brk (gifts from Chris Rushlow), UAS-babo ${ }^{C A}$ (gift from Michael O'Connor), elav-Gal80 (30) (gift from Lily Yeh Jan and Yuh-Nung Jan), $d l g^{40-2}$ (gift from David Bilder). Stocks that were obtained from the Bloomington Drosophila Stock Center: UAS-InR ${ }^{A C T}$ (\#8263),dpp-Gal4/TM6B (\#1553) UAS-dpp (\#1486), UAS-tkv ${ }^{C A}$ (\#36537), brk-GFP.FPTB (\#38629), dilp8 ${ }^{\text {MI00727 }}$ (\#33079), Aug21-Gal4 (\#30137), AKH-Gal4 (\#25684), UAS-dcr2 (\#24650), UAS-dpp RNAi (\#25782, \#33618, \#36779), r4-Gal4 (\#33832), UAS-preproANF-EMD (\#7001), G-TRACE-3 (\#28281), UAS-Mad ${ }^{\text {RNAi }}$ (\#40397), UAS-Med ${ }^{R N A i}$ (\#19688), AbdB-Gal4 (\#55848), UAS-tdTom (\#36328), UAS-tdGFP (\#35836).

\section{Immunohistochemistry and microscopy}

Larvae were dissected in PBS, fixed 20 min in 4\% PFA, permeabilized with $0.1 \%$ Triton X-100, and blocked with $10 \%$ normal goat serum. Primary antibodies used are: rabbit anti-Smad3 (Abcam \#52903, 1:500), rabbit anti-Sad (1:250), rabbit anti-Phm (1:250), rabbit anti-Dib (1:250) and guinea pig anti-Spok (1:1000) (gifts from Michael O'Connor), rabbit anti-Ptth (1:100) (gift from Pierre Léopold), mouse anti-Armadillo N2 7A1 (31)(DSHB, 1:100), mouse anti-Dlg 4F3 (32)(DHSB, 1:100), rabbit anti-GFP (Torrey Pines Biolabs \#TP401, 1:500), mouse anti-GFP (Abcam AB290, 1:500), Cambridge, MA), rabbit anti-cleaved DCP-1 (Cell Signaling Asp216, 1:250), rabbit anti-FOXO (1:1000) (gift from Michael Thomas Marr) (33). Secondary antibodies used are: goat anti-mouse 555 (Invitrogen \#A32727), goat anti-mouse 647 (Invitrogen \#A32728), goat anti-rabbit 555 (Invitrogen \#A32732), goat anti-rabbit 647 (Invitrogen \#A32733) goat anti-guinea pig 555 (Invitrogen \#A-21435) (all, 1:500) as well as phalloidin-TRITC (Sigma \#P1951, 1:500) and DAPI 
(Invitrogen \#D1306, 1:500). Samples were mounted in SlowFade Gold (Invitrogen \#S36937) and imaged on a Zeiss 700 LSM confocal microscope.

\section{Developmental timing assay and $r n^{t s}>$ temperature shift experiments}

Fertilized eggs were collected on grape juice plates for $4 \mathrm{~h}$. L1 stage larvae were transferred onto standard Bloomington food supplemented with yeast paste at a density of 50 animals per vial. For constitutive expression without the presence of a temperature-sensitive Gal80, animals were raised consistently at $25^{\circ} \mathrm{C}$ and pupal counts were taken every $8 \mathrm{~h}$. Three independent experiments were conducted for each condition. $r n^{t s}>$ animals were raised at $18^{\circ} \mathrm{C}$ until day 7 (early third instar), then transferred to $30^{\circ} \mathrm{C}$ for a $24 \mathrm{~h}$ temperature shift and subsequently returned to $18^{\circ} \mathrm{C}$. Pupal counts were taken every $12 \mathrm{~h}$. Three independent experiments were conducted for each condition.

\section{0-Hydroxyecdysone feeding}

A stock solution of $10 \mathrm{mg} / \mathrm{ml}$ 20E (Sigma \#H5142) in ethanol was prepared. 50 larvae per vial were raised on standard Bloomington food. At $72 \mathrm{~h} \mathrm{AEL,} \mathrm{1mg} \mathrm{20-Hydroxyexcdysone} \mathrm{or}(100 \mu \mathrm{l}$ stock solution or ethanol for controls) was added to each vial.

\section{Quantification of wing discs and pupal weight}

Pupae from developmental timing assays were collected at the pharate adult stage were cleaned with $70 \%$ ethanol, dried and weighed in groups of 30 in three or four independent experiments. GraphPad Prism 6 was used to determine statistical significance between groups by one-way ANOVA using Tukey's or Dunnett's test. Pupae were placed on double-sided adhesive tape for imaging using a Leica transmitted light microscope (TL RCl, Germany). Adobe Photoshop was used to quantify the area of imaginal disc confocal images dissected from larvae from developmental timing assays.

\section{Hemolymph extraction}

Larvae were bled into chilled PBS supplemented with protease inhibitor (Roche \# 11697498001) on a cold aluminum block using a fine tungsten needle to puncture the cuticle. 


\section{Western Blotting}

For homogenates, staged larvae at $72 \mathrm{~h} \mathrm{AEL}$ were transferred into chilled buffer containing $35 \mathrm{mM}$ Tris- $\mathrm{HCl} \mathrm{pH} 6.8,129 \mathrm{mM} \mathrm{NaCl}, 4 \mathrm{mM} \mathrm{KCl}, 2 \mathrm{mM} \mathrm{CaCl}_{2}$ supplemented with protease inhibitor (Roche \#11697498001). Larvae were homogenized for $1 \mathrm{~min}$ with a microtube homogenizer, followed by centrifugations for $5 \mathrm{~min}$ at $16,000 \mathrm{~g}$ and $2 \mathrm{~min}$ at $16,000 \mathrm{~g}$ at $4^{\circ} \mathrm{C}$. Homogenates or hemolymph were boiled for $10 \mathrm{~min}$ in Laemmli sample buffer, run on 10\% Mini-Protean TGX gels (Bio-Rad) and transferred to nitrocellulose membrane (Bio-Rad). Primary antibody used was rabbit anti-GFP (Torrey Pines Biolabs \#TP401, 1:1000). Protein bands were detected with secondary antibody HRP anti-rabbit (Santa Cruz Biotechnology \#sc-2030, 1:2500) and Western Lightning Plus-ECL (PerkinElmer \#NEL103001EA).

\section{Ex vivo organ culture}

20 brain-ring gland complexes were dissected from wandering third instar Oregon $\mathrm{R}$ larvae in Schneider's medium (Gibco \# 21720024) by pulling mouth hooks from which salivary glands, lymph gland and fat body was removed. Complexes were subsequently co-cultured with either wing imaginal discs or hemolymph from $r n^{t s}>d p p$ larvae larvae in Schneider's medium supplemented with 10\% FBS (Invitrogen \#26140087) and Penicillin-Streptomycin at 1:100 of a 5,000 U/ml stock (Gibco \#15070063).

\section{Irradiation}

Density controlled third instar larvae were placed on shallow food plates and irradiated with $45 \mathrm{~Gy}$ in an X-ray cabinet (Faxitron, Tucson, AZ), followed by dissection after $12 \mathrm{~h}$.

\section{Heat-shock clone induction}

Flies with UAS transgenes were crossed to either ywhsFIp;;Act>>Gal4,UAS-GFP or ywhsFlp;;Act $>>$ Gal4,UAS-RFP and raised at $25^{\circ} \mathrm{C}$. Larvae were staged and density controlled as described for developmental timing assay, then heat shocked in a $37^{\circ} \mathrm{C}$ water bath for 5 min at 24 or $48 \mathrm{hAEL}$ before returning to $25^{\circ} \mathrm{C}$ until dissection. 

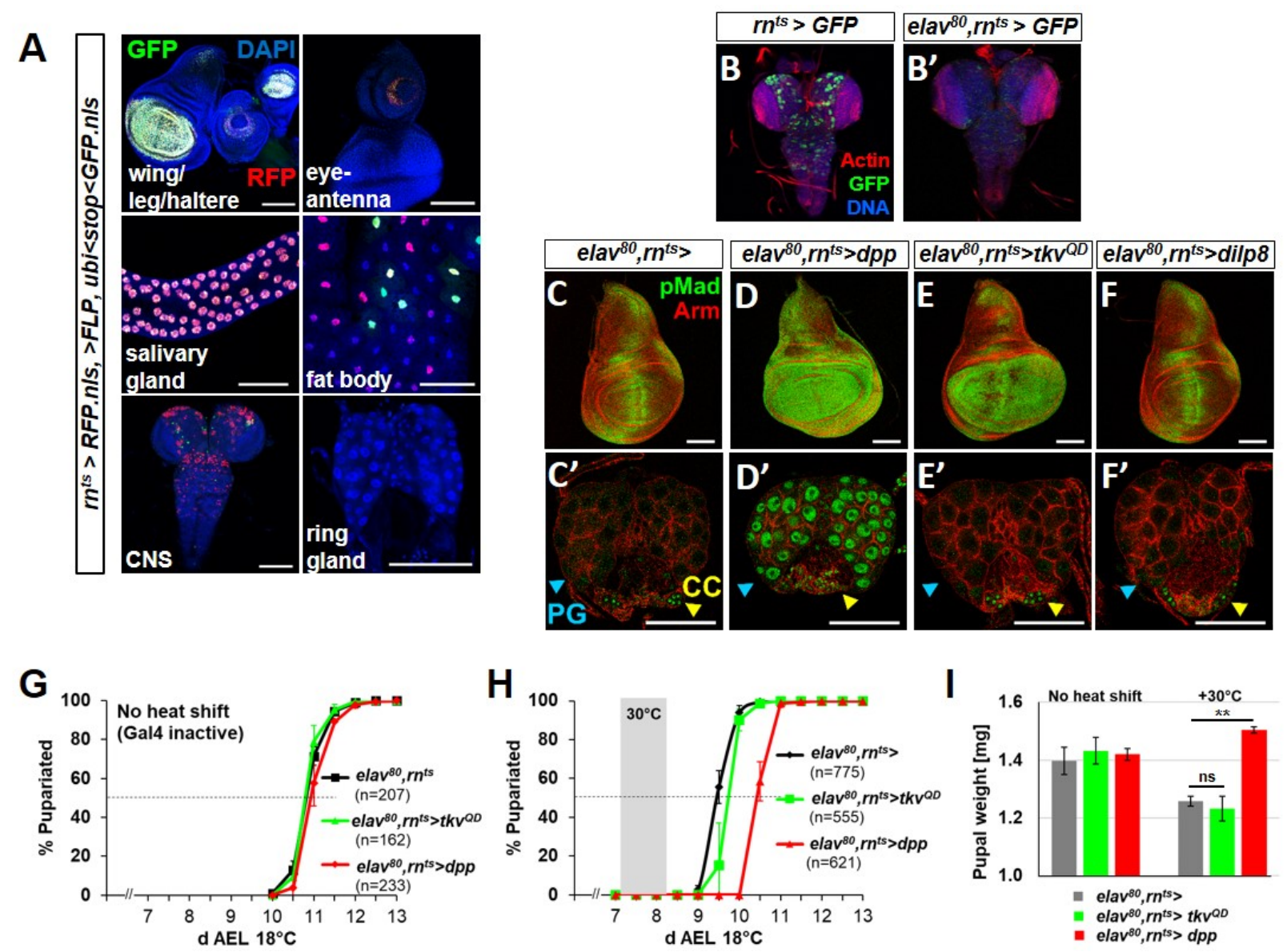

Supplementary Figure S1: Characterization of $r n^{t s}>$ expression and investigation of the role of expression in the central nervous system (CNS) on pupariation delay

(A) Characterization of expression of $r n^{t s}>$ using G-TRACE (16). RFP (red) indicates recent expression, GFP (green) indicates either recent or past expression. Expression is observed in imaginal discs and the salivary glands, in scattered nuclei in the fat body and in the central nervous system (CNS) i.e. brain and ventral nerve cord. No expression was detected in any portion of the ring gland.

(B, $\mathbf{B}^{\prime}$ ) Expression of $r n^{t s}>$ GFP in the CNS in the absence (B) or presence (B') of elav-Gal80 $\left(e l a v^{80}\right)$.

(C-F, ' ' $-\mathbf{F}^{\prime}$ ) Effect of reducing CNS expression of $r n^{\text {ts }}>$ using elav-Gal80 on Dpp signaling in wing discs (C-F) and the ring gland $\left(\mathbf{C}^{\prime}-\mathbf{F}^{\prime}\right)$. Unlike in the PG (blue arrowhead), pMad is observed in the CC (yellow arrowhead) in all genotypes.

(G, H) Timing of pupariation of different genotypes cultured at $18^{\circ} \mathrm{C}$ either without (G) or with (H) a shift to $30^{\circ} \mathrm{C}$ for $24 \mathrm{~h}$ beginning on day $7 \mathrm{AEL}$. Delays when compared to elav $v^{80}, \mathrm{rn}^{t s}>$ are: elav ${ }^{80}, r n^{t s}>d p p 28.4 \pm 6.4 \mathrm{~h} ; e l a v^{80}, r n^{t s}>t k v^{Q D} 8.0 \pm 1.2 \mathrm{~h}$

(I) Pupal weights of the genotypes shown in (G) and (H). These are, without a heat shift, elav ${ }^{80}$ $r n^{t s}>1.40 \pm 0.05 \mathrm{mg}$; elav ${ }^{80}, r n^{t s}>t k v^{Q D} 1.43 \pm 0.05 \mathrm{mg} ;$ elav $^{80}, r n^{t s}>d p p 1.42 \pm 0.02 \mathrm{mg}(\mathrm{n}=90$ per genotype) and with a heat shift elav ${ }^{80} r n^{t s}>1.26 \pm 0.02 \mathrm{mg}$; elav ${ }^{80}, r n^{t s}>t k v^{Q D} 1.23 \pm 0.04 \mathrm{mg}$; elav ${ }^{80}, r n^{t s}>d p p 1.50 \pm 0.01 \mathrm{mg}$ ( $\mathrm{n}=90$ per genotype). Error bars indicate standard deviations. $\mathrm{ns}$ indicates not significant; ${ }^{* *}$ indicates $p<0.01$. All scale bars $=100 \mu \mathrm{m}$. 

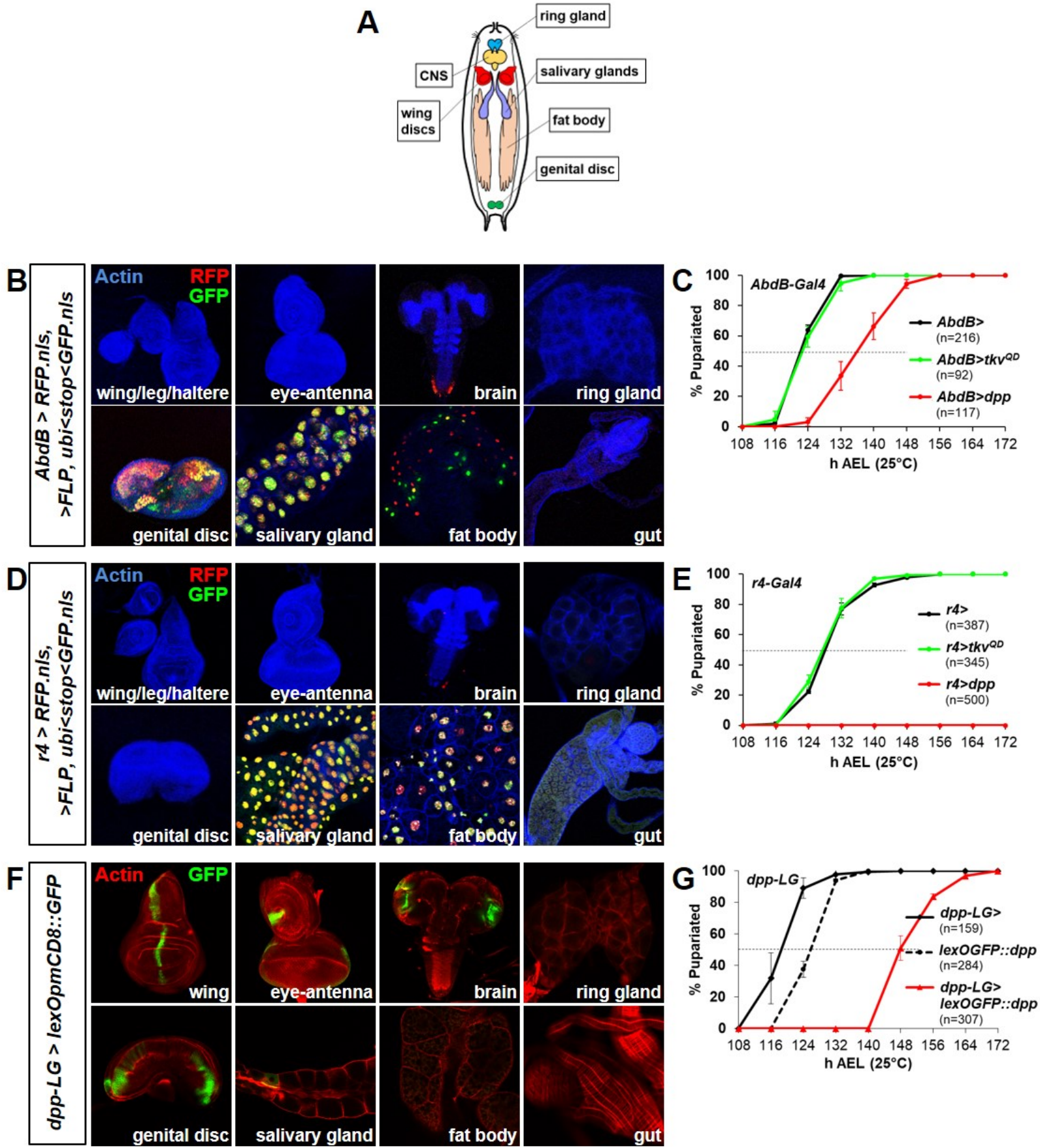

Supplementary Figure S2: Effects of expression of $d p p$ and $t k v^{Q D}$ in a variety of tissues on the timing of pupariation.

(A) Cartoon of larva showing tissues relevant to experiments in (B-G) (B-G) Effect of expressing $d p p$ or $t k v^{Q D}$ using heterologous expression systems on pupariation timing. The expression patterns of $A b d B-G a l 4$ (B), r4-Gal4 (D) are shown using G-TRACE and of $d p p-L G$ using a lexOp-mCD8::GFP (F). The corresponding graphs (C, E, G) show the effect on the timing of pupariation. 

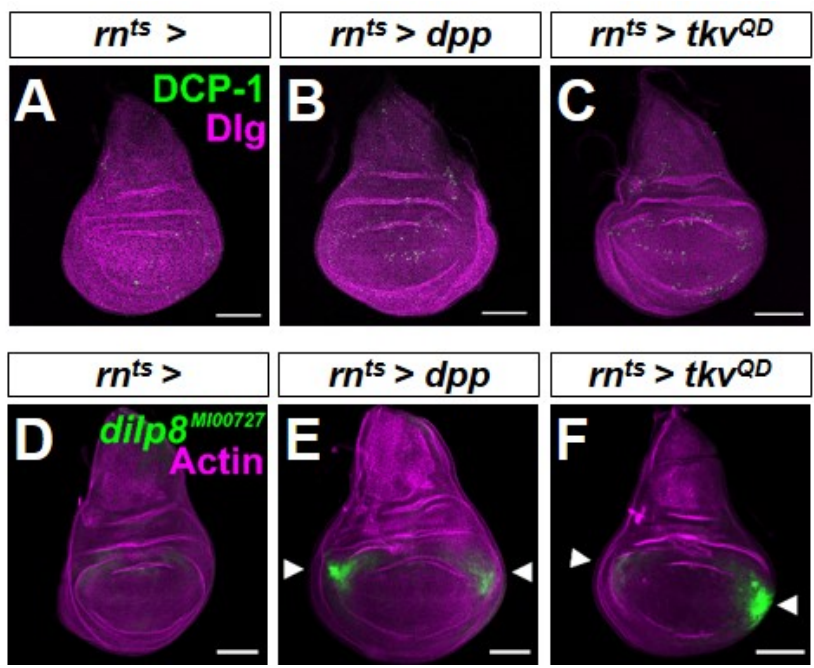

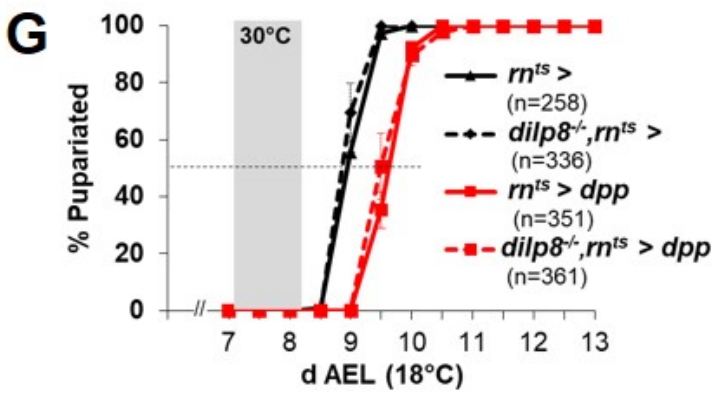

Supplementary Figure S3: $r n^{t s}>$ expression of $d p p$ or $t k v^{Q D}$ in wing discs causes relatively little cell death or dilp8 expression.

(A-C) Effect of increased expression of $d p p$ or $t k v^{Q D}$ on levels of apoptosis (visualized by antiDCP-1). Anti-Dlg labels cell outlines.

(D-F) Effect of increased expression of $d p p$ or $t k v^{Q D}$ on expression of a GFP gene trap in the dilp8 gene (11). Arrowheads indicate increased dilp8 expression in lateral regions of the wing pouch suggestive of mild overgrowth in this region. Phalloidin (actin) labels cells in the disc. Scale bars are $100 \mu \mathrm{m}$.

(G) Comparison of the delay in pupariation elicited by $r n^{t s}>d p p$ in a wild-type and dilp8 homozygous mutant background. The time course of pupariations of $r n^{t s}>$ (no UAS-dpp) in the two backgrounds are also shown. Mean pupariation times are $r n^{t s}>8.93 \pm 0.03 \mathrm{~d} ; r n^{t s}>d p p$ $9.62 \pm 0.03 \mathrm{~d}$ AEL (delay = 16.4 $\pm 0.3 \mathrm{~h}$ ); dilp8 -/-, $r n^{t s}>8.87 \pm 0.03 \mathrm{~d}$; dilp8 -/-, $r n^{t s}>d p p 9.62 \pm 0.11 \mathrm{~d}$ AEL (delay $=18.00 \pm 0.52 \mathrm{~h}$ ). 

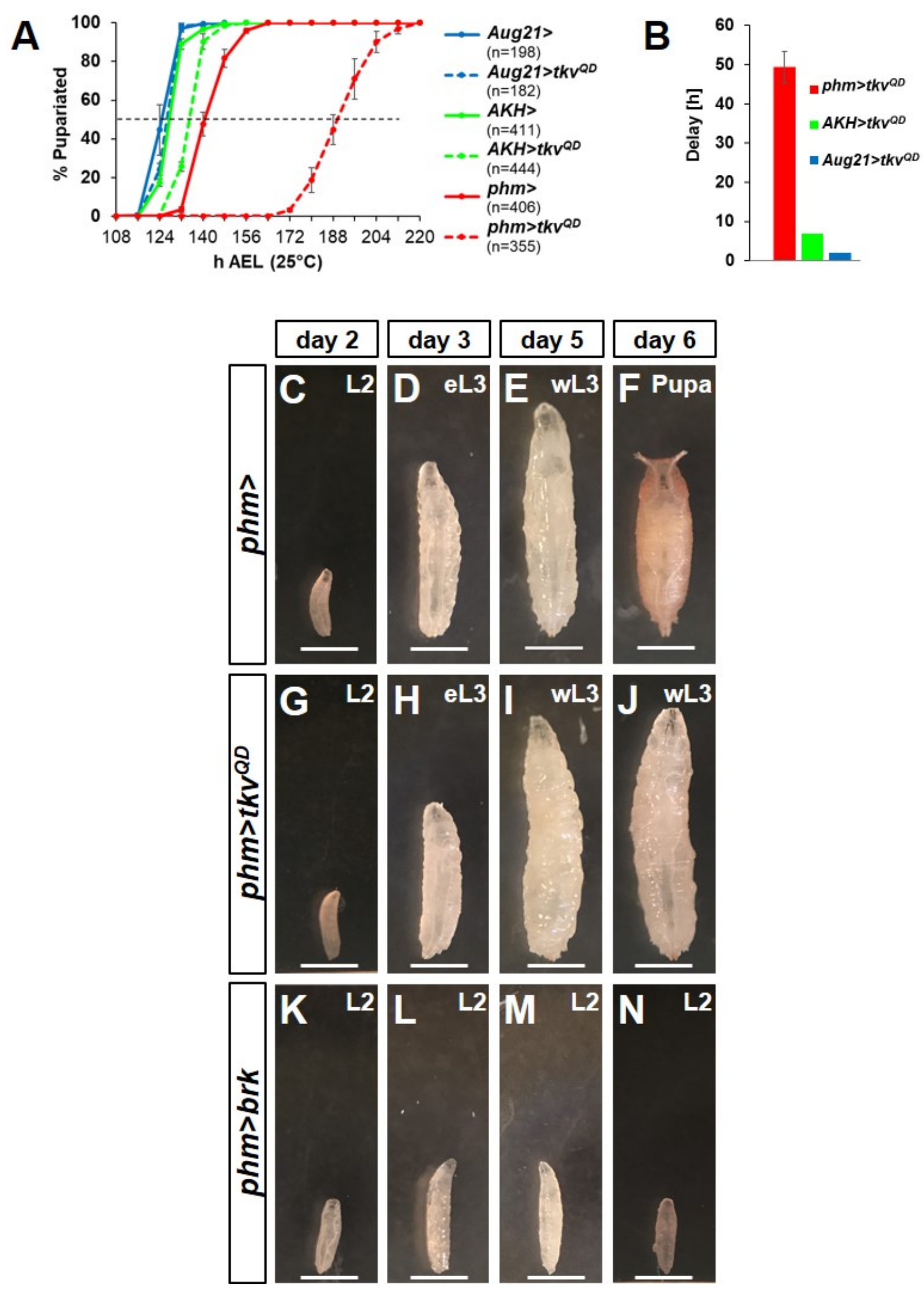
Supplementary Figure S4: Effects of altering Dpp signaling or of brinker (brk) expression in the PG on pupariation

$(\mathrm{A}, \mathrm{B})$ Effect of expressing $t k v^{Q D}$ in different parts of the ring gland on pupariation timing. phmGal4 drives expression in the PG (34); AKH-Gal4 (35) is expressed in the CC; Aug21-Gal4 is expressed in the CA (36). Mean pupariation times are: phm>140.3 $\pm 1.5 \mathrm{~h} ;$ phm $>t k v^{Q D} 189.7 \pm 2.5$

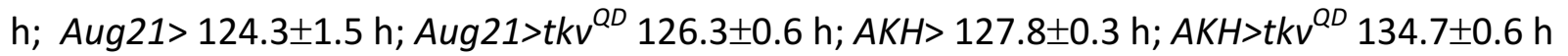
The delays shown in (B) show the effect of including UAS-tkv ${ }^{Q D}$ when compared to the corresponding Gal4 driver line alone. Error bars are standard deviations.

(C-N) Temporal comparison of phm> (C-F), phm>tkv ${ }^{Q D}$ (G-J) and phm>brk (K-N) larvae. Images of stage- and density-controlled animals raised at $25^{\circ} \mathrm{C}$. On day $2 \mathrm{AEL}$, (C) control phm> animals, (G) $p h m>t k v^{Q D}$ and (K) phm $>b r k$ animals are at the second instar stage (L2). phm> and phm $>t k v^{Q D}$ animals progress to the early third instar (eL3) on day $3(\mathbf{D}, \mathbf{H})$ and wandering third instar ( $\mathrm{WL}$ ) on day $5(\mathrm{E}, \mathrm{I}) . p h m>$ animals pupariate by day $6(\mathbf{F})$ while $p h m>t k v^{Q D}$ animals remain in $w L 3(\mathrm{~J})$ until a much delayed pupariation on day 8 or day 9. phm $>$ brk animals do not progress to eL3 on day 3 (L) and remain small and in second instar through days 5 and $6(\mathbf{M}, \mathbf{N})$. However, while still in L2, they exhibit wandering behavior for multiple days followed by lethality without pupariation. Wandering behavior in L2 is reminiscent of the early pupariation phenotype observed with expression of a constitutively-active form of the Activin receptor, Baboon (7). Scale bars are $1 \mathrm{~mm}$. 

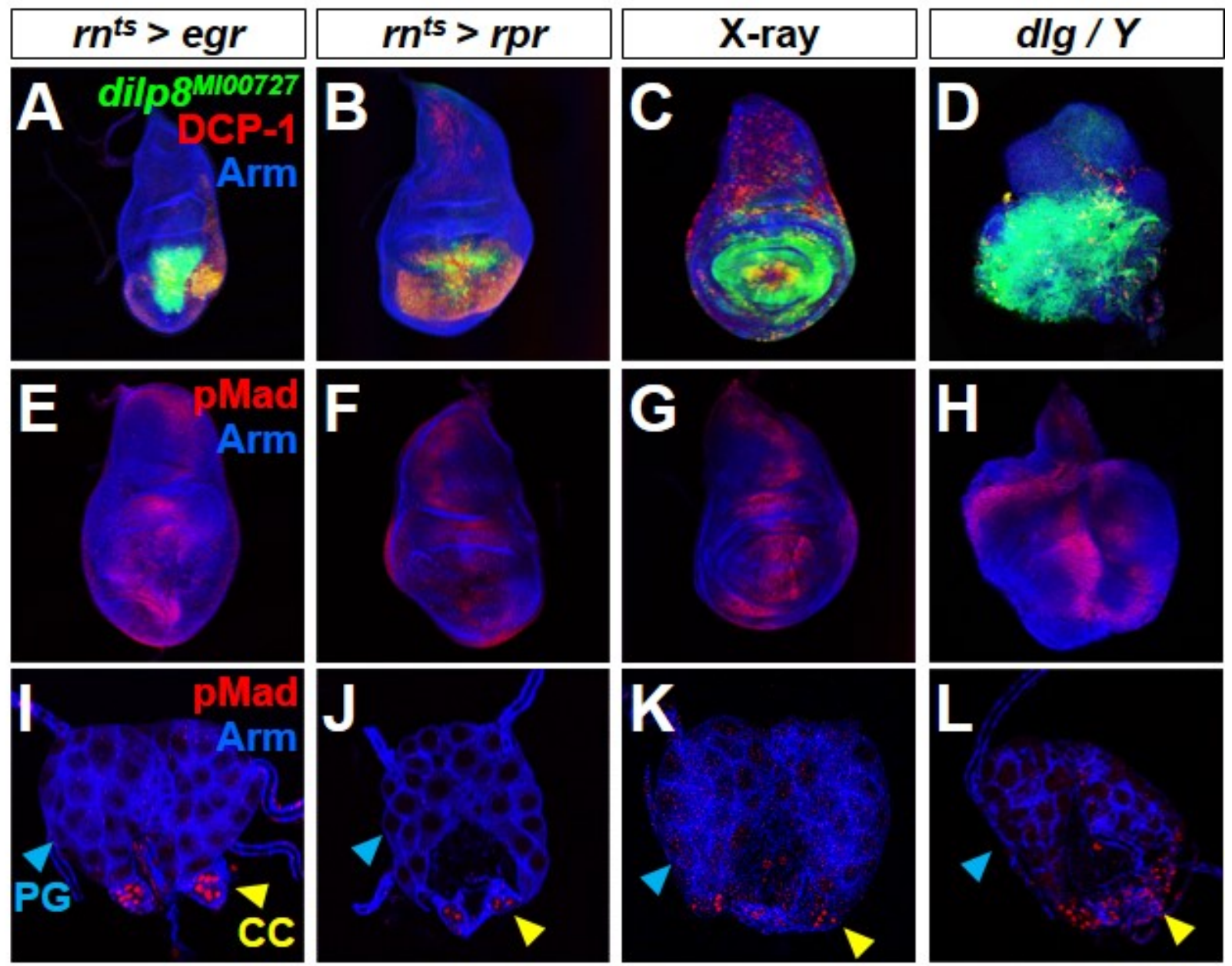

Supplementary Figure S5: Cell death and disc overgrowth result in dilp8 expression in discs but not in ectopic Dpp signaling in either the disc or ring gland.

Wing discs $(\mathbf{A}-\mathbf{H})$ and ring glands (I-L) shown under conditions that increase cell death by expression of eiger (egr) $(\mathbf{A}, \mathbf{E}, \mathbf{I})$, reaper $(\operatorname{rpr})(\mathbf{B}, \mathbf{F}, \mathbf{J})$, or $\mathbf{X}$-ray irradiation $(\mathbf{C}, \mathbf{G}, \mathbf{K})$ or neoplastic overgrowth of the disc in $d / g$ hemizygotes $(\mathbf{D}, \mathbf{H}, \mathbf{L})$. Each of these conditions caused dilp8 expression in the disc but did not result in ectopic nuclear pMad either in the wing disc or PG. The CC cells have nuclear pMad in all genotypes. 

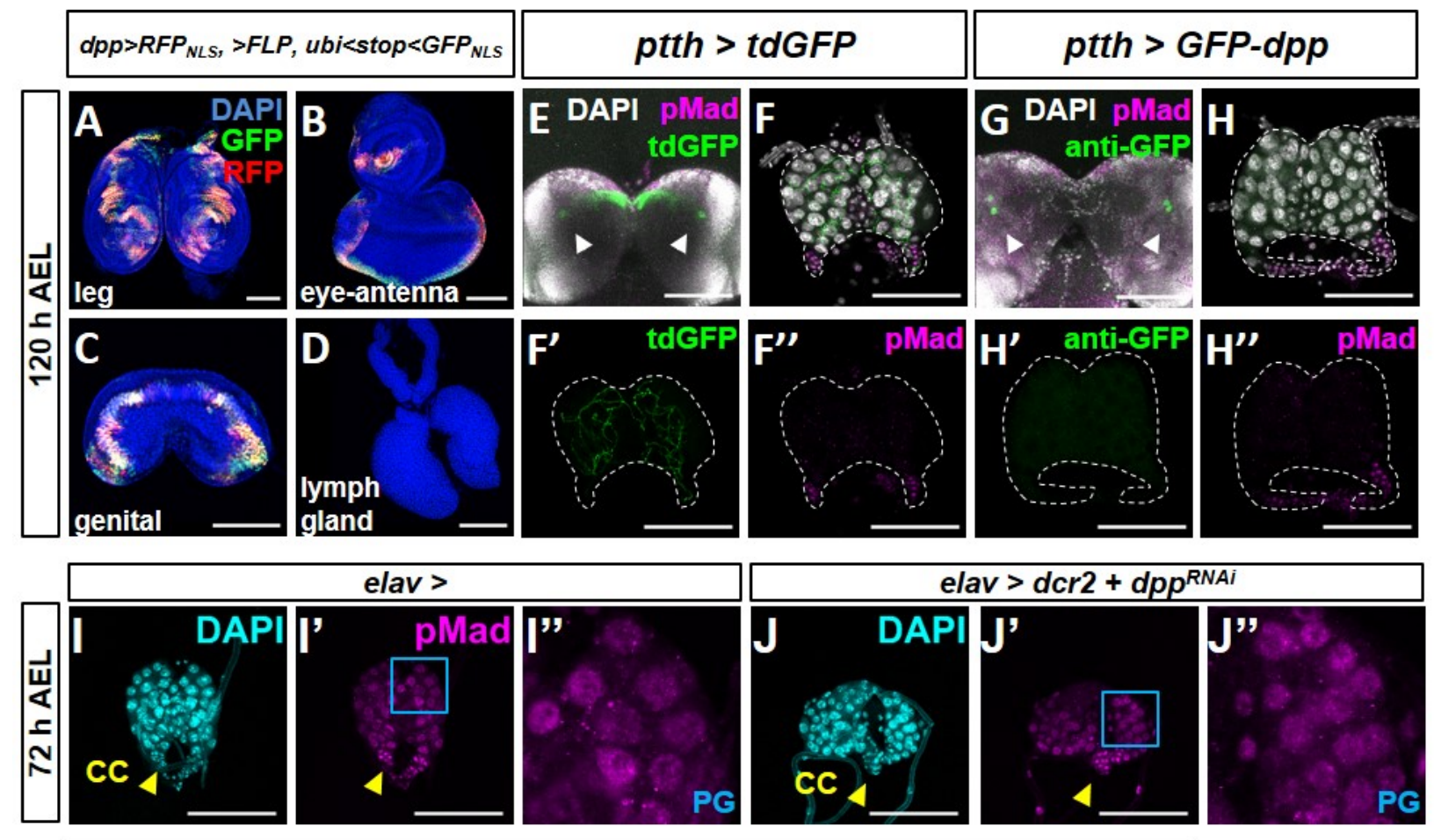

elav > RFP.n/s, >FLP, ubi<stop<GFP.n/s
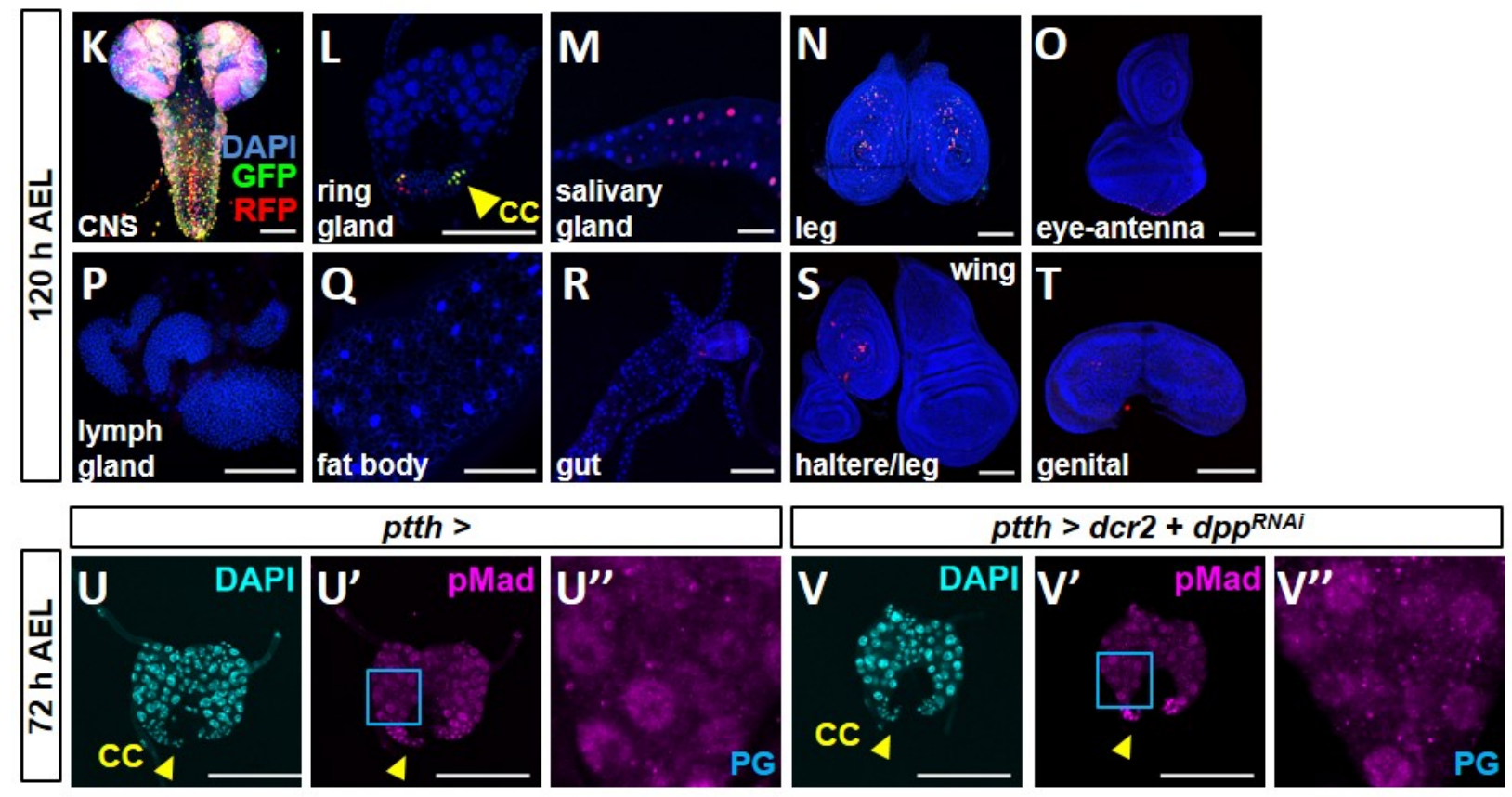

Supplementary Figure S6: Further characterization of the source of Dpp that reaches the PG in L3 larvae

(A-D) Additional images from G-TRACE experiments (16) to characterize expression of dpp-Gal4. Expression is observed in leg discs (A), eye-antennal disc (B), genital disc (C) but not in the lymph gland (D). 
A previous study (37) reported the expression of a dpp-lacZ reporter in the CA of the ring gland. In that same study, $d p p$ expression in the ring gland, measured using quantitative RT-PCR from total RNA prepared from ring glands, was reported to increase in late $L 3$, peaking in wandering L3 larvae. These observations are not easy to reconcile with our finding that Dpp signaling in the PG decreases during L3. We have not observed any pMad gradient in the PG based on the distance of cells from the CA. We have also not observed any current or past expression of $d p p$ Gal4 in the ring gland using G-TRACE (Fig. 3T) or UAS-tdTom (Fig. 3U). LG-dpp, lexOpmCDB::GFP is also not expressed in the ring gland (Fig. S2F). Since $d p p-G a / 4$ is not expressed in the ring gland and $d p p$-Gal4 UAS-dcr2, UAS-dpp ${ }^{R N A i}$ can reduce pMad expression in the PG, the source of Dpp that acts on the PG is unlikely to be in the ring gland itself.

(E, F-F”, G, H-H") Effect of expressing GFP-dpp in the PTTH neurons. Expression of tdGFP using ptth-Gal4 which labels cell membranes $\left(\mathbf{E}, \mathbf{F}, \mathbf{F}^{\prime}\right)$ shows axons that innervate the PG but does not result in nuclear pMad in the PG. GFP is visualized using GFP fluorescence. Expression using GFP-dpp using ptth-Gal4 (G, $\left.\mathbf{H}, \mathbf{H}^{\prime}\right)$ does not result in delivery of GFP-Dpp to the PG $\left(\mathbf{G}, \mathbf{H}, \mathbf{H}^{\prime}\right)$ or an increase in nuclear pMad in the PG (H"). In (G, $\left.\mathbf{H},-\mathbf{H}^{\prime \prime}\right)$, anti-GFP antibody was added to detect even low levels of GFP expression. Arrowheads indicate PTTH neurons.

(I-I", J-J") Effect of elav-Gal4, UAS-dpp ${ }^{R N A i}$ in ring glands dissected at $72 \mathrm{~h}$ AEL. Nuclear pMad is detected both in elav-Gal4 (I-I") and elav-Gal4, UAS-dcr2, UAS-dpp ${ }^{R N A i}$ (J-J") cells in the PG. (K-T) Characterization of expression of the elav-Gal4 driver using G-TRACE. Note expression in the brain and ventral nerve cord (K), CC cells in the ring gland (L), salivary gland (M), leg disc (N) and eye-antennal disc ( $\mathbf{O})$. No expression in lymph gland $(\mathbf{P})$, fat body $(\mathbf{Q})$, gut $(\mathbf{R})$, wing and haltere discs $(\mathbf{S})$, and genital disc $(\mathbf{T})$.

(U-V") Effect of ptth-Gal4, UAS-dpp ${ }^{R N A i}$ in ring glands dissected at $72 \mathrm{~h}$ AEL. Nuclear pMad is detected both in ptth-Gal4 (U, U', U') and ptth-Gal4, UAS-dcr2, UAS-dpp ${ }^{R N A i}\left(\mathbf{V}, \mathbf{V}^{\prime}, \mathbf{V}^{\prime \prime}\right)$ cells in the PG. Scale bars are $100 \mu \mathrm{m}$. 

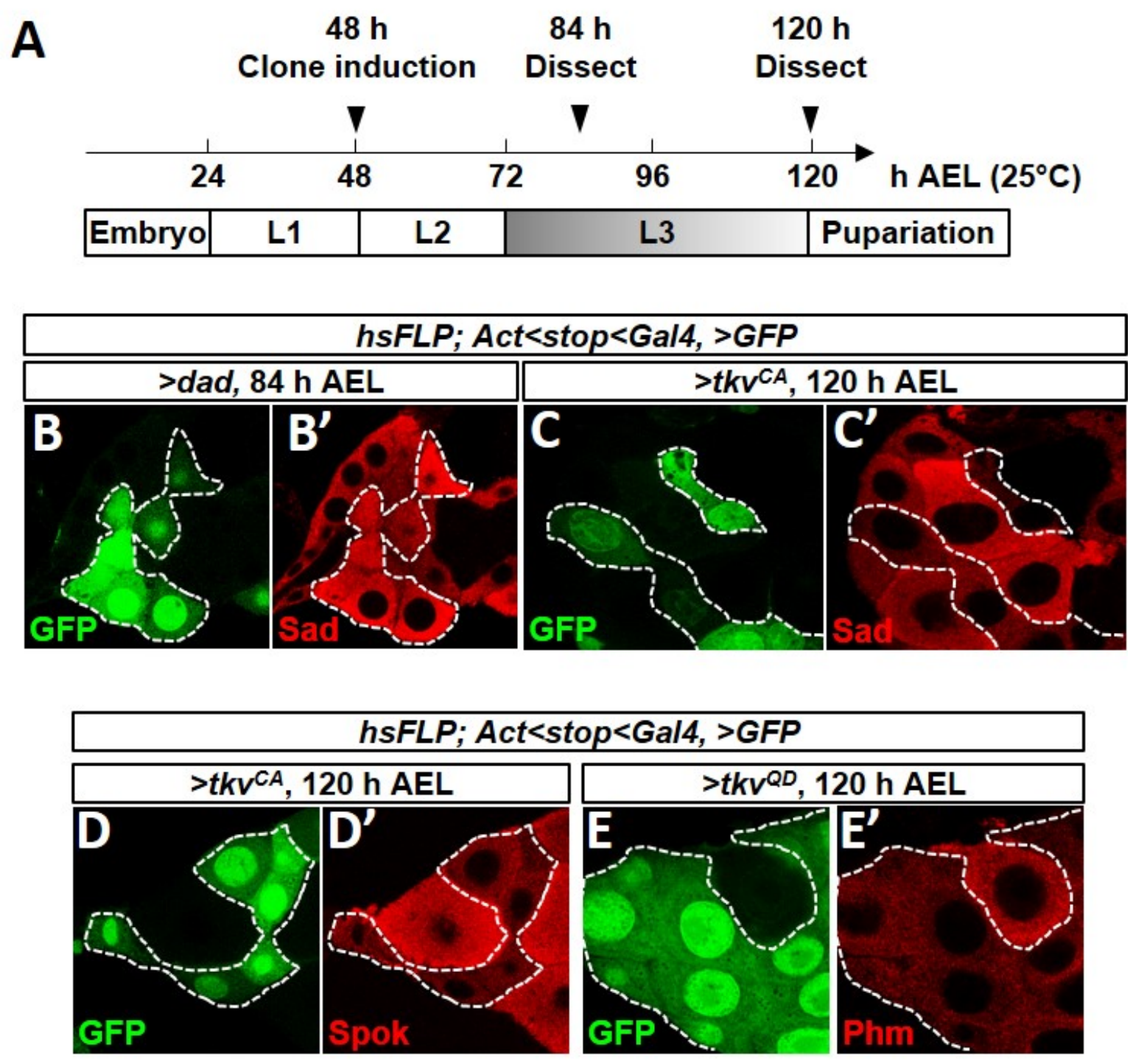

Supplementary Figure S7: Further characterization of regulation of ecdysone biosynthesis enzymes by Dpp signaling

(A) Schematic of heat-shock clone induction experiments. Cultures were maintained at $25^{\circ} \mathrm{C}$. FLP-out clones (act<stop<Gal4) were induced $48 \mathrm{~h}$ AEL. FLP-out clones in the PG show uneven expression of GFP possibly because of asynchrony of or variability in the number of endocycles between the individual polyploid cells of the PG.

(B, B') Cell-autonomous reduction in Dpp signaling in $84 \mathrm{~h} \mathrm{AEL} \mathrm{PG} \mathrm{cells} \mathrm{using} \mathrm{UAS-dad} \mathrm{induces}$ upregulation of Shadow (Sad), although the effect is less obvious than with Disembodied (Dib) (shown in Figure 4). GFP-positive cells express UAS-dad.

(C-E, C'-E') Cell-autonomous activation of the Dpp pathway in $120 \mathrm{~h}$ AEL PG cells by expressing UAS transgenes that express an activated form of Tkv (UAS-tkv ${ }^{Q D}$ in $E ; U A S-t k v^{C A}$ in $C$ and D) causes a reduction in levels of Shadow (Sad) $\left(\mathbf{C}, \mathbf{C}^{\prime}\right)$, Spookier (Spok) (D, D'), and Phantom (Phm) $\left(E, E^{\prime}\right)$. GFP-positive cells express UAS-tkv ${ }^{Q D}$. Once again, the effect on Sad expression is less than with the other enzymes. 

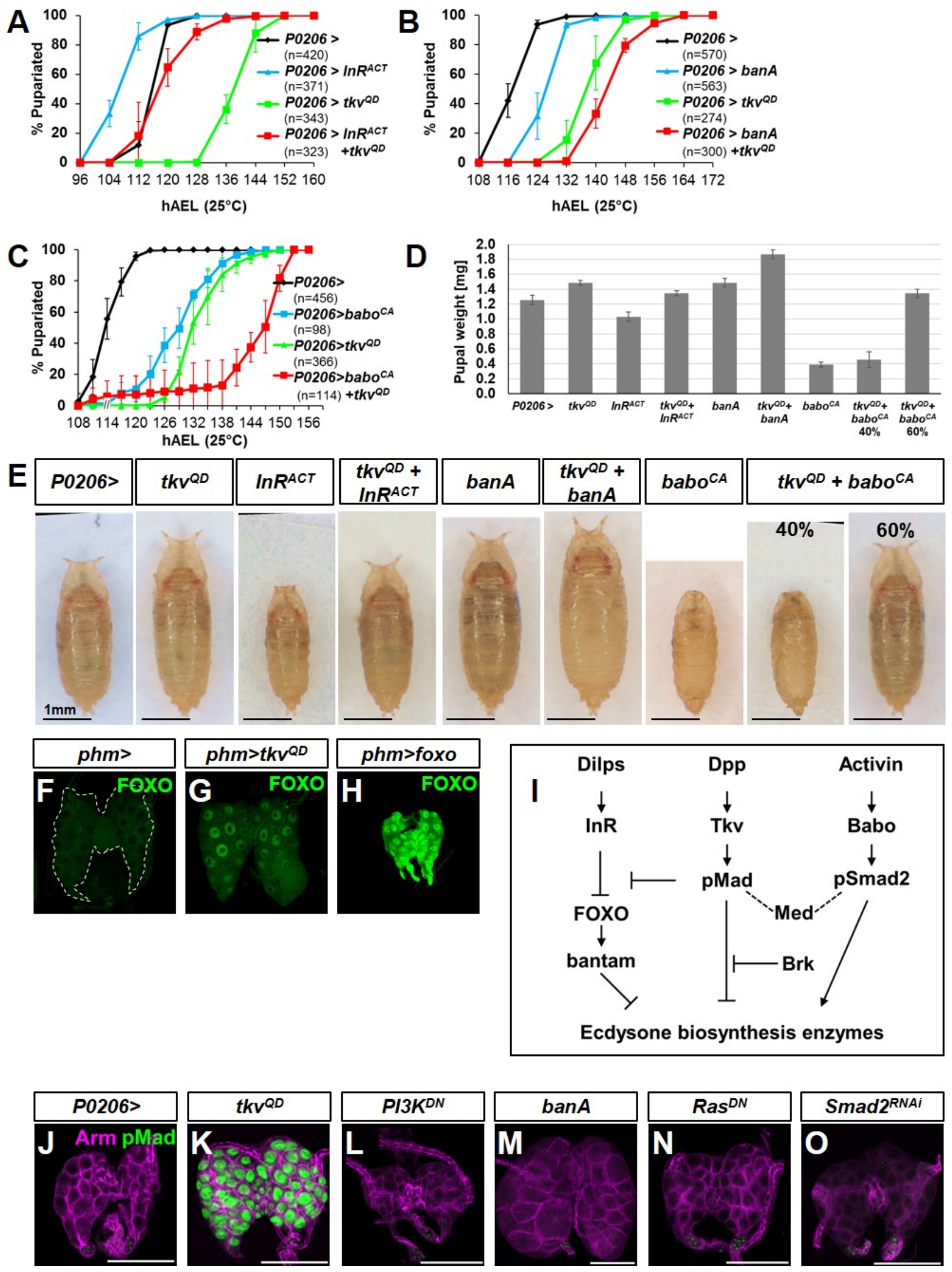


\section{Supplementary Figure S8: Dpp signaling acts along with other pathways that regulate ecdysteroidogenesis in the PG}

(A-E) Activation of different signaling pathways in the ring gland using P0206-Gal4.

$(\mathbf{A}, \mathbf{D}, \mathrm{E})$ Interaction of Dpp signaling with the insulin pathway in the PG. (A) UAS-tkv ${ }^{Q D}$ delays pupariation. Expression of an activated form of the insulin receptor (UAS-InR ${ }^{A C T}$ ) accelerates pupariation. With concurrent expression of both transgenes, pupariation timing is similar to that of the P0206-Gal4 driver alone. Pupariation times were: $P 0206>115.8 \pm 0.3 \mathrm{~h}$; $P 0206>t k v^{Q D} 140.7 \pm 7.2 \mathrm{~h} ; P 0206>\ln R^{A C T} 106.3 \pm 1.3 \mathrm{~h} ; P 0206>t k v^{Q D}+\ln R^{A C T} 117.0 .7 \pm 3.0 \mathrm{~h}$. Similarly, $P 0206>\operatorname{In} R^{A C T}$ pupae are small, $P 0206>t k v^{Q D}$ pupae are large and $P 0206>\operatorname{In} R^{A C T}+$ $t k v^{Q D}$ pupae are similar in size or weight to $P 0206>$ pupae $(D, E)$.

(B, D, E) Interaction of Dpp signaling with ban. UAS-banA also delays pupariation. Pupariation times were $P 0206>117.0 \pm 1.0 \mathrm{~h} ; P 0206>t k v^{Q D} 138.0 \pm 2.6 \mathrm{~h} ; P 0206>$ banA $125.7 \pm 1.5 \mathrm{~h}$; $P 0206>t k v^{Q D}+$ banA $143.0 \pm 1.7 \mathrm{~h}$. Thus, co-expression of UAS-tkv ${ }^{Q D}$ and UAS-banA delays pupariation more than either transgene alone. Since the delay elicited by UAS-banA overexpression is less than that obtained with $U A S-t k v^{Q D}$, it is unlikely that $t k v^{Q D}$ delays pupariation exclusively by increasing ban expression. Similarly, the increase in pupal size or weight obtained with co-expression of UAS-tkv ${ }^{Q D}$ and UAS-ban is greater than the effect obtained with either transgene alone (D, E). Note that even though the pupariation delay obtained with UAS-banA is less than that obtained with UAS-tkv ${ }^{Q D}$, the effects on pupal size are similar suggesting that UAS-banA is more effective than $U A S-t k v^{Q D}$ in increasing growth rate. (C, D, E) Interaction of Dpp signaling with Activin signaling. Pupariation times were P0206> $114.5 \pm 1.3 \mathrm{~h} ; P 0206>t k v^{Q D} 129.2 \pm 1.5 \mathrm{~h} ; P 0206>b a b o^{C A} 133.5 \pm 3.1 \mathrm{~h} ; P 0206>t k v^{Q D}+b a b o^{C A}$ $148.0 \pm 3.0 \mathrm{~h}$. Expression of a constitutively-active form of the Activin receptor (UAS-babo ${ }^{C A}$ ) delayed development. However, the larvae appeared delayed in $\mathrm{L} 2$ generating small pupae as has been described previously (7). With co-expression of $t k v^{Q D}$ and $b a b o^{C A}$, approximately $40 \%$ of the pupae resembled $\mathrm{P0206}>b a b o^{C A}$ pupae in appearance and stage-precocious pupariation in L3. The remaining $60 \%$ resembled pupae that had gone through the $L 3$ stage and formed pharate adults. The delay in pupariation was close to being additive of the delays caused by either transgene alone. It is possible that the combination of Dpp and Activin signaling leads to an either/or phenotype due to a competition of pMad and pSmad2 with Medea (22).

(D) Pupal weights of all of the conditions shown in panels (A-C). Summary of tests of statistical significance of pairwise comparisons of pupal weight measurements.

(E) Images of pupae of all the conditions shown in panels $(A-C)$.

(F-H) FOXO protein visualized in ring glands using anti-FOXO antibody. FOXO is cytoplasmic in phm > ring glands (G) and nuclear in phm>FOXO ring glands (H). However, in $20 \%$ of phm>tkv ${ }^{Q D}$ ring glands we observed nuclear FOXO (G). The reason for the incomplete penetrance of this phenomenon is unclear. A similar frequency of nuclear FOXO was observed when using two different transgenes that encode activated $t k v$ and also when using the P0206-Gal4 instead of phm-Gal4. Thus Dpp signaling could impact the insulin pathway upstream of FOXO.

(I) Model for interaction of Dpp pathway with the InR/FOXO/bantam and Activin signaling. (J-O) Ring glands dissected at 120hAEL. (J) P0206>; (K) P0206>tkv ${ }^{Q D}$; (L) P0206>PI3K ${ }^{D N}$; (M) P0206>banA; (N) P0206>Ras ${ }^{D N}$ (0) P0206>Smad2 ${ }^{R N A i}$. Other than P0206>tkv ${ }^{Q D}$, none of these other transgenes, that have each been reported to cause a delay in pupariation (K-N), causes 
nuclear pMad accumulation in the PG indicating that these pathways do not activate Dpp signaling in the PG. Scale bars are $100 \mu \mathrm{m}$.

Table showing all pairwise comparisons for pupal weights in panel (D):

\begin{tabular}{|c|c|}
\hline $\begin{array}{l}\text { Tukey's multiple comparisons test } \\
\text { (pupal weight of each genotype vs. each other } \\
\text { genotype) }\end{array}$ & Summary \\
\hline$P 0206>$ vs. $P 0206>t k v^{Q D}$ & *** \\
\hline$P 0206>$ vs. $P 0206>\ln R^{A C T}$ & $* * *$ \\
\hline$P 0206>$ vs. $P 0206>\ln R^{A C T}+t k v^{Q D}$ & ns \\
\hline$P 0206>$ vs. $P 0206>b a b o^{C A}$ & $* * * *$ \\
\hline$P 0206>$ vs. $P 0206>b a b o^{C A}+t k v^{Q D} 40 \%$ & $* * * *$ \\
\hline$P 0206>$ vs. $P 0206>b a b o^{C A}+t k v^{Q D} 60 \%$ & ns \\
\hline$P 0206>$ vs. $P 0206>$ banA & $* * *$ \\
\hline$P 0206>$ vs. $P 0206>$ banA+tkv ${ }^{Q D}$ & $* * \star *$ \\
\hline$P 0206>t k v^{Q D}$ vs. $P 0206>\ln R^{A C T}$ & $* * * *$ \\
\hline$P 0206>t k v^{Q D}$ vs. $P 0206>\ln R^{A C T}+\mathrm{tkv}^{\mathrm{QD}}$ & ns \\
\hline$P 0206>t k v^{Q D}$ vs. $P 0206>$ babo $^{\text {CA }}$ & $* * * *$ \\
\hline$P 0206>t k v^{Q D}$ vs. $P 0206>b^{b a b o}{ }^{C A}+\mathrm{tkv}^{Q D} 40 \%$ & $* * * *$ \\
\hline$P 0206>t k v^{Q D}$ vs. $P 0206>$ babo $^{C A}+\mathrm{tkv}^{\mathrm{QD}} 60 \%$ & ns \\
\hline$P 0206>t k v^{Q D}$ vs. $P 0206>$ banA & ns \\
\hline$P 0206>t k v^{Q D}$ vs. $P 0206>b a n A+t k v^{Q D}$ & $* * * *$ \\
\hline$P 0206>\ln R^{A C T}$ vs. $P 0206>\ln R^{A C T}+t k v^{Q D}$ & $* * * *$ \\
\hline$P 0206>\ln R^{A C T}$ vs. $P 0206>b a b o^{C A}$ & $* * * *$ \\
\hline$P 0206>\ln R^{A C T}$ vs. $P 0206>$ babo $^{C A}+\mathrm{tkv}^{\mathrm{QD}} 40 \%$ & $* * * *$ \\
\hline$P 0206>\ln R^{A C T}$ vs. $P 0206>$ babo $^{C A}+\mathrm{tkv}^{Q D} 60 \%$ & **** \\
\hline$P 0206>\ln R^{A C T}$ vs. $P 0206>$ banA & $* * \star *$ \\
\hline$P 0206>\ln R^{A C T}$ vs. $P 0206>$ banA+tkv ${ }^{Q D}$ & $* * * *$ \\
\hline$P 0206>\ln R^{A C T}+t k v^{Q D}$ vs. $P 0206>b a b o^{C A}$ & $* * * *$ \\
\hline$P 0206>\ln R^{A C T}+t k v^{Q D}$ vs. $P 0206>b a b o^{C A}+t k v^{Q D} 40 \%$ & $* * * *$ \\
\hline$P 0206>\ln R^{A C T}+t k v^{Q D}$ vs. $P 0206>b a b o^{C A}+t k v^{Q D} 60 \%$ & ns \\
\hline$P 0206>\ln R^{A C T}+t k v^{Q D}$ vs. $P 0206>$ banA & ns \\
\hline$P 0206>\ln R^{A C T}+t k v^{Q D}$ vs. $P 0206>b a n A+t k v^{Q D}$ & $* * * *$ \\
\hline$P 0206>b a b o^{C A}$ vs. $P 0206>b a b o^{C A}+t k v^{Q D} 40 \%$ & ns \\
\hline$P 0206>b^{2} a b o^{C A}$ vs. $P 0206>$ babo $^{C A}+\mathrm{tkv}^{Q D} 60 \%$ & $* * * *$ \\
\hline$P 0206>$ babo ${ }^{C A}$ vs. $P 0206>$ banA & $* * * *$ \\
\hline$P 0206>b a b o^{C A}$ vs. $P 0206>b a n A+t k v^{Q D}$ & $* * * *$ \\
\hline
\end{tabular}




\begin{tabular}{|c|c|}
\hline $\begin{array}{l}P 0206>b a b o^{C A}+t k v^{Q D} 40 \% \text { vs. } P 0206>b a b o^{C A}+t k v^{Q D} \\
60 \%\end{array}$ & $* * * *$ \\
\hline$P 0206>b a b o^{C A}+t k v^{Q D} 40 \%$ vs. $P 0206>b a n A$ & $* * * *$ \\
\hline$P 0206>b a b o^{C A}+t k v^{Q D} 40 \%$ vs. $P 0206>b a n A+t k v^{Q D}$ & $* * * *$ \\
\hline$P 0206>b a b o^{C A}+t k v^{Q D} 60 \%$ vs. $P 0206>b a n A$ & ns \\
\hline$P 0206>b a b o^{C A}+t k v^{Q D} 60 \%$ vs. $P 0206>b a n A+t k v^{Q D}$ & $* * * *$ \\
\hline$P 0206>$ banA vs. $P 0206>$ banA $+t k v^{Q D}$ & $* * * *$ \\
\hline
\end{tabular}

\begin{tabular}{|l|l|}
\hline Summary & P value \\
\hline ns & $\geq 0.05$ \\
\hline$*$ & 0.01 to 0.05 \\
\hline$* *$ & 0.001 to 0.01 \\
\hline$* * *$ & 0.0001 to 0.001 \\
\hline$* * * *$ & $<0.0001$ \\
\hline
\end{tabular}




\section{REFERENCES}

1. N. Yamanaka, K. F. Rewitz, M. B. O'Connor, Ecdysone control of developmental transitions: lessons from Drosophila research. Annual review of entomology 58, 497-516 (2013).

2. L. Boulan, M. Milan, P. Leopold, The Systemic Control of Growth. Cold Spring Harb Perspect Biol 7, (2015).

3. B. C. Stieper, M. Kupershtok, M. V. Driscoll, A. W. Shingleton, Imaginal discs regulate developmental timing in Drosophila melanogaster. Dev Biol 321, 18-26 (2008).

4. K. F. Rewitz, N. Yamanaka, L. I. Gilbert, M. B. O'Connor, The insect neuropeptide PTTH activates receptor tyrosine kinase torso to initiate metamorphosis. Science 326, 14031405 (2009).

5. J. Colombani et al., Antagonistic actions of ecdysone and insulins determine final size in Drosophila. Science 310, 667-670 (2005).

6. C. Mirth, J. W. Truman, L. M. Riddiford, The role of the prothoracic gland in determining critical weight for metamorphosis in Drosophila melanogaster. Curr Biol 15, 1796-1807 (2005).

7. Y. Y. Gibbens, J. T. Warren, L. I. Gilbert, M. B. O'Connor, Neuroendocrine regulation of Drosophila metamorphosis requires TGFbeta/Activin signaling. Development 138, 26932703 (2011).

8. L. Boulan, D. Martin, M. Milan, bantam miRNA promotes systemic growth by connecting insulin signaling and ecdysone production. Curr Biol 23, 473-478 (2013).

9. J. Rodenfels et al., Production of systemically circulating Hedgehog by the intestine couples nutrition to growth and development. Genes Dev 28, 2636-2651 (2014).

10. J. Colombani, D. S. Andersen, P. Leopold, Secreted peptide Dilp8 coordinates Drosophila tissue growth with developmental timing. Science 336, 582-585 (2012).

11. A. Garelli, A. M. Gontijo, V. Miguela, E. Caparros, M. Dominguez, Imaginal discs secrete insulin-like peptide 8 to mediate plasticity of growth and maturation. Science 336,579 582 (2012).

12. F. Hamaratoglu, M. Affolter, G. Pyrowolakis, Dpp/BMP signaling in flies: from molecules to biology. Semin Cell Dev Biol 32, 128-136 (2014).

13. D. Nellen, R. Burke, G. Struhl, K. Basler, Direct and long-range action of a DPP morphogen gradient. Cell 85, 357-368 (1996).

14. E. V. Entchev, A. Schwabedissen, M. Gonzalez-Gaitan, Gradient formation of the TGFbeta homolog Dpp. Cell 103, 981-991 (2000).

15. S. Rao, C. Lang, E. S. Levitan, D. L. Deitcher, Visualization of neuropeptide expression, transport, and exocytosis in Drosophila melanogaster. J Neurobiol 49, 159-172 (2001).

16. C. J. Evans et al., G-TRACE: rapid Gal4-based cell lineage analysis in Drosophila. Nat Methods 6, 603-605 (2009).

17. R. D. St Johnston, W. M. Gelbart, Decapentaplegic transcripts are localized along the dorsal-ventral axis of the Drosophila embryo. EMBO J 6, 2785-2791 (1987).

18. J. B. Brown et al., Diversity and dynamics of the Drosophila transcriptome. Nature 512, 393-399 (2014). 
19. T. E. Haerry, O. Khalsa, M. B. O'Connor, K. A. Wharton, Synergistic signaling by two BMP ligands through the SAX and TKV receptors controls wing growth and patterning in Drosophila. Development 125, 3977-3987 (1998).

20. Q. Ou, K. King-Jones, What goes up must come down: transcription factors have their say in making ecdysone pulses. Curr Top Dev Biol 103, 35-71 (2013).

21. K. Tsuneizumi et al., Daughters against dpp modulates dpp organizing activity in Drosophila wing development. Nature 389, 627-631 (1997).

22. A. J. Peterson, M. B. O'Connor, Activin receptor inhibition by Smad2 regulates Drosophila wing disc patterning through BMP-response elements. Development 140, 649-659 (2013).

23. O. Wartlick et al., Dynamics of Dpp signaling and proliferation control. Science 331, 1154-1159 (2011).

24. S. Harmansa, I. Alborelli, D. Bieli, E. Caussinus, M. Affolter, A nanobody-based toolset to investigate the role of protein localization and dispersal in Drosophila. eLife 6, (2017).

25. M. Ma, X. Cao, J. Dai, J. C. Pastor-Pareja, Basement Membrane Manipulation in Drosophila Wing Discs Affects Dpp Retention but Not Growth Mechanoregulation. Dev Cell 42, 97-106 e104 (2017).

26. J. C. Pastor-Pareja, T. Xu, Shaping cells and organs in Drosophila by opposing roles of fat body-secreted Collagen IV and perlecan. Dev Cell 21, 245-256 (2011).

27. R. K. Smith-Bolton, M. I. Worley, H. Kanda, I. K. Hariharan, Regenerative growth in Drosophila imaginal discs is regulated by Wingless and Myc. Dev Cell 16, 797-809 (2009).

28. R. Yagi, F. Mayer, K. Basler, Refined LexA transactivators and their use in combination with the Drosophila Gal4 system. Proc Natl Acad Sci U S A 107, 16166-16171 (2010).

29. J. Brennecke, D. R. Hipfner, A. Stark, R. B. Russell, S. M. Cohen, bantam encodes a developmentally regulated microRNA that controls cell proliferation and regulates the proapoptotic gene hid in Drosophila. Cell 113, 25-36 (2003).

30. C. H. Yang et al., Control of the postmating behavioral switch in Drosophila females by internal sensory neurons. Neuron 61, 519-526 (2009).

31. B. Riggleman, P. Schedl, E. Wieschaus, Spatial expression of the Drosophila segment polarity gene armadillo is posttranscriptionally regulated by wingless. Cell 63, 549-560 (1990).

32. D. Parnas, A. P. Haghighi, R. D. Fetter, S. W. Kim, C. S. Goodman, Regulation of postsynaptic structure and protein localization by the Rho-type guanine nucleotide exchange factor dPix. Neuron 32, 415-424 (2001).

33. O. Puig, M. T. Marr, M. L. Ruhf, R. Tjian, Control of cell number by Drosophila FOXO: downstream and feedback regulation of the insulin receptor pathway. Genes Dev 17, 2006-2020 (2003).

34. H. Ono et al., Spook and Spookier code for stage-specific components of the ecdysone biosynthetic pathway in Diptera. Dev Biol 298, 555-570 (2006).

35. G. Lee, J. H. Park, Hemolymph sugar homeostasis and starvation-induced hyperactivity affected by genetic manipulations of the adipokinetic hormone-encoding gene in Drosophila melanogaster. Genetics 167, 311-323 (2004).

36. G. Adam, N. Perrimon, S. Noselli, The retinoic-like juvenile hormone controls the looping of left-right asymmetric organs in Drosophila. Development 130, 2397-2406 (2003). 
bioRxiv preprint doi: https://doi.org/10.1101/180562; this version posted August 24,2017 . The copyright holder for this preprint (which was not certified by peer review) is the author/funder. All rights reserved. No reuse allowed without permission.

37. J. Huang et al., DPP-mediated TGFbeta signaling regulates juvenile hormone biosynthesis by activating the expression of juvenile hormone acid methyltransferase. Development 138, 2283-2291 (2011). 